\title{
EXACT CONTROLLABILITY AND STABILIZABILITY OF THE KORTEWEG-DE VRIES EQUATION
}

\author{
DAVID L. RUSSELL AND BING-YU ZHANG
}

ABstract. In this paper, we consider distributed control of the system described by the Korteweg-de Vries equation

$$
\partial_{t} u+u \partial_{x} u+\partial_{x}^{3} u=f
$$

on the interval $0 \leq x \leq 2 \pi, t \geq 0$, with periodic boundary conditions

$$
\partial_{x}^{k} u(2 \pi, t)=\partial_{x}^{k} u(0, t), \quad k=0,1,2,
$$

where the distributed control $f \equiv f(x, t)$ is restricted so that the "volume" $\int_{0}^{2 \pi} u(x, t) d x$ of the solution is conserved. Both exact controllability and stabilizibility questions are studied for the system.

In the case of open loop control, if the control $f$ is allowed to act on the whole spatial domain $(0,2 \pi)$, it is shown that the system is globally exactly controllable, i.e., for given $T>0$ and functions $\phi(x), \psi(x)$ with the same "volume", one can alway find a control $f$ so that the system (i)-(ii) has a solution $u(x, t)$ satisfying

$$
u(x, 0)=\phi(x), \quad u(x, T)=\psi(x) .
$$

If the control $f$ is allowed to act on only a small subset of the domain $(0,2 \pi)$, then the same result still holds if the initial and terminal states, $\psi$ and $\phi$, have small "amplitude" in a certain sense.

In the case of closed loop control, the distributed control $f$ is assumed to be generated by a linear feedback law conserving the "volume" while monotonically reducing $\int_{0}^{2 \pi} u(x, t)^{2} d x$. The solutions of the resulting closed loop system are shown to have uniform exponential decay to a constant state. As in the open loop control case, a small amplitude assumption is needed if the control is allowed to act on only a small subdomain.

The smoothing property of the periodic (linear) KdV equation discovered recently by Bourgain has played an important role in establishing the exact controllability and stabilizability results presented in this paper.

\section{INTRODUCTION}

In the present work we consider distributed control of a class of equations which may be described as being of Korteweg-de Vries (KdV) type. These have the general form

$$
\partial_{t} u+\alpha u \partial_{x} u+\partial_{x}^{3} u=f
$$

Received by the editors May 12, 1994.

1991 Mathematics Subject Classification. Primary 35K60, 93C20.

Supported in part by NSF Grant DMS-9402838. Reproduction in whole or in part is permitted for U.S. Government purposes. 
with $\alpha$ a real number. For $\alpha=0$ this is the third order linear dispersion equation studied by the authors in [15]. All cases $\alpha \neq 0$ are essentially equivalent, as we see in [9], and therefore we here assume, for simplicity, that $\alpha=1$.

The distributed control case consists in a study of the equation (1.1) in which $f \equiv f(x, t)$, with possible restrictions on the form of that function which we will indicate as we proceed, appears as the control function by which solutions are to be influenced. We study this system on the interval $0 \leq x \leq 2 \pi, t \geq 0$, with periodic boundary conditions

$$
\partial_{x}^{k} u(2 \pi, t)=\partial_{x}^{k} u(0, t), \quad k=0,1,2,
$$

so that $x=2 \pi$ is effectively identified with $x=0$, yielding a spatial domain equivalent to a circle.

To explain the control problem to be studied, a certain amount of background is required. For appropriately smooth solutions of the unforced equation $(f(x, t) \equiv 0)$ on the indicated domain with periodic boundary conditions (1.2), and extensions of those boundary conditions as required to express further smoothness, it is known [9], [10] that there is an infinite set of conserved integral quantities of which only the first three are of interest to us here:

$$
\begin{gathered}
\int_{0}^{2 \pi} u(x, t) d x \\
\int_{0}^{2 \pi} u(x, t)^{2} d x \\
\int_{0}^{2 \pi}\left(\partial_{x} u(x, t)\right)^{2}-\frac{1}{3} u(x, t)^{3} d x .
\end{gathered}
$$

From the historical origins [9], [12] of the KdV equation, involving the behavior of water waves in a shallow channel, it is natural to think of (1.3) as expressing conservation of volume or mass. That context suggests that the control problem might also be appropriately studied in a volume conserving context. For solutions of (1.1) continuously differentiable with respect to $t$ and three times continuously differentiable with respect to $x$ we have

$$
\begin{aligned}
\partial_{t} \int_{0}^{2 \pi} & u(x, t) d x=\int_{0}^{2 \pi}\left(-u(x, t) \partial_{x} u(x, t)-\partial_{x}^{3} u(x, t)+f(x, t)\right) d x \\
& =\int_{0}^{2 \pi}\left\{\partial_{x}\left(-\frac{1}{2} u(x, t)^{2}-\partial_{x}^{2} u(x, t)\right)+f(x, t)\right\} d x \\
& =\int_{0}^{2 \pi} f(x, t) d x,
\end{aligned}
$$

from which we conclude that volume is conserved in the control system (1.1) just in case, as we assume henceforth,

$$
\int_{0}^{2 \pi} f(x, t) d x \equiv 0 .
$$

A more interesting case is obtained if some further a priori restrictions are imposed on the applied control $f(x, t)$. Let us suppose that $g(x)$ is a smooth 
function defined for $x \in[0,2 \pi]$ such that

$$
2 \pi[g]=\int_{0}^{2 \pi} g(x) d x=1
$$

where $[g]$ denotes the mean value of the function $g$ over the interval $(0,2 \pi)$. We further restrict attention to controls of the form

$$
f(x, t)=G h:=g(x)\left(h(x, t)-\int_{0}^{2 \pi} g(y) h(y, t) d y\right) ;
$$

thus $h=h(x, t)$ may be considered as the new control function. It is easily seen that

$$
\int_{0}^{2 \pi} f(x, t) d x \equiv 0
$$

for $f$ of the form (1.8); therefore the restriction (1.6) is satisfied for all controls of the form (1.8).

Depending on the support of the function $g(x)$ in the domain $[0,2 \pi]$, there are two different control situations. If the support of the function $g$ is the whole interval $[0,2 \pi]$, then our control acts on the whole domain and we refer to it as global control. If the support of the function $g$ is a proper subset of the interval $[0,2 \pi]$, our control acts only on a subdomain and we refer to it as local control. Obviously we have more control power in the global control situation than in the local control case. On the other hand, the local control situation includes more cases of practical interest and is therefore more relevant in general.

Our main concerns in this paper are exact controllability and stabilizability of the system (1.1), (1.2), (1.8).

In order to describe our main results precisely, we introduce some preliminary material at this point.

Let

$$
\phi_{k}(x)=\frac{1}{\sqrt{2 \pi}} e^{i k x}, \quad k=0, \pm 1, \pm 2, \ldots
$$

Then $\left\{\phi_{k}\right\}_{k=-\infty}^{\infty}$ forms an orthonormal basis in the space $L^{2}(0,2 \pi)$. We may define the Sobolev space $H_{p}^{s}(0,2 \pi)$ of order $s(s \geq 0)$ as the space of all real $2 \pi$-periodic functions

$$
v(x)=\sum_{k=-\infty}^{+\infty} v_{k} \phi_{k}(x)
$$

such that

$$
\left\{\sum_{k=-\infty}^{+\infty}\left|v_{k}\right|^{2}(1+|k|)^{2 s}\right\}^{1 / 2}<+\infty .
$$

The left hand side of (1.9) is a Hilbert norm for $H_{p}^{s}(0,2 \pi)$; we denote it by $\|v\|_{s}$.

We now describe the main results of this paper.

For the control system just introduced the exact control problem consists in using the indicated control function to transfer the system, during $[0, T]$, between a given initial state $u(x, 0)=u_{0}(x)$ and a given terminal state $u(x, T)=u_{1}(x)$, in an appropriate function space of system states, necessarily, in view of the volume conserving control actions under consideration, such that

$$
\int_{0}^{2 \pi} u_{0}(x) d x=\int_{0}^{2 \pi} u_{1}(x) d x .
$$


In the global control case, the control $h$ acts on the whole domain $[0,2 \pi]$ and we have the following strong controllability result.

Theorem 1.1. Let $T>0$ and $s \geq 1$ be given and assume that the function $g$ in (1.8) satisfies

$$
|g(x)|>\beta>0, \quad \forall x \in[0,2 \pi] .
$$

Then for any $u_{0}, u_{1} \in H_{p}^{s}(0,2 \pi)$ with $\left[u_{0}\right]=\left[u_{1}\right]$, there exists a control function $h \in L^{2}\left([0, T] ; H_{p}^{s-1}(0,2 \pi)\right)$ such that the equation

$$
\partial_{t} u+u \partial_{x} u+\partial_{x}^{3} u=G h
$$

has a solution $u \in C\left([0, T] ; H_{p}^{s}(0,2 \pi)\right)$ satisfying

$$
u(x, 0)=u_{0}(x), \quad u(x, T)=u_{1}(x) .
$$

In other words we have "global" exact controllability in the global control case.

In the local control case, the support of the function $g$ may be a very small part of the domain $[0,2 \pi]$; thus our control power is quite limited. In this situation, we have the following "local" exact controllability result.

Theorem 1.2. Let $T>0$ and $s \geq 0$ be given. Then there exists a $\delta>0$ such that for any $u_{0}, u_{1} \in H_{p}^{s}(0,2 \pi)$ with $\left[u_{0}\right]=\left[u_{1}\right]$ and

$$
\left\|u_{0}\right\|_{s} \leq \delta, \quad\left\|u_{1}\right\|_{s} \leq \delta,
$$

one can find a control $h \in L^{2}\left([0, T] ; H_{p}^{s}(0,2 \pi)\right)$ such that the equation

$$
\partial_{t} u+u \partial_{x} u+\partial_{x}^{3} u=G h
$$

has a solution $u \in C\left([0, T] ; H_{p}^{s}(0,2 \pi)\right)$ satisfying

$$
u(x, 0)=u_{0}(x), \quad u(x, T)=u_{1}(x) .
$$

It is familiar that each control problem has associated with it a corresponding stabilization problem in which the control is required, for each $t$, to be a functional of fixed form (i.e., independent of $t$ ) of the system state $u(\cdot, t)$ and the objective of the control action is to cause the system to approach, as $t \rightarrow \infty$, a particular set of equilibrium states. As explained in more detail for the linear case in [15], the stabilization problem in our current context has as its goal that the controlled solution $u(x, t)$ should tend, as $t \rightarrow \infty$, to the constant state $\bar{u}(x) \equiv\left[u_{0}\right]$. To achieve this goal for the system (1.1), (1.2) and (1.8), we may employ, for some $\mathcal{K}>0$, the following feedback control law:

$$
h(x, t)=-\mathcal{K} \operatorname{sign}(g(x)) u(x, t) ;
$$

we obtain the closed loop system, studied earlier in [8] and [19],

$$
\left\{\begin{array}{l}
\partial_{t} u+u \partial_{x} u+\partial_{x}^{3} u=-\mathcal{K} G u, \quad u(x, 0)=u_{0}(x), \\
u(0, t)=u(2 \pi, t), \quad \partial_{x} u(0, t)=\partial_{x} u(2 \pi, t), \quad \partial_{x}^{2} u(0,2 \pi)=\partial_{x}^{2} u(2 \pi, t),
\end{array}\right.
$$

where the function $g(x)$ in the definition of the operator $G$ is then assumed to be nonnegative.

In the global control case where the function $g \equiv 1$, it has been shown in [8] that the solution $u(x, t)$ decays to the mean value of its initial data exponentially in the space $H_{p}^{n}(0,2 \pi)$ if $u_{0} \in H_{p}^{n}(0,2 \pi)(n \geq 2)$. 
In the local control case, as explained earlier, feedback control may act on only a small part of the domain $[0,2 \pi]$; thus the control power is limited. In this situation we have the following asymptotic stability result for small amplitude solutions of the system (1.15).

Theorem 1.3. Let $\mathcal{K}>0$ and $s=0$ or $s \geq 1$ be given. There exist positive constants $M, \delta$ and $\gamma$ such that if $u_{0} \in H_{p}^{s}(0,2 \pi)$ with $\left\|u_{0}-\left[u_{0}\right]\right\|_{s} \leq \delta$, then the corresponding solution $u$ of the system (1.15) satisfies

$$
\left\|u(\cdot, t)-\left[u_{0}\right]\right\|_{s} \leq M e^{-\gamma t}\left\|u_{0}-\left[u_{0}\right]\right\|_{s}
$$

for any $t \geq 0$, so that small amplitude solutions $u$ decay at a uniform exponential rate to the corresponding constant state $\left[u_{0}\right]$ with respect to the norm in the space $H_{p}^{s}(0,2 \pi)$ as $\rightarrow \infty$.

The paper is organized as follows. In Section 2, we consider a linear system associated with the system (1.1), (1.2), (1.8),

$$
\left\{\begin{array}{l}
\partial_{t} v+\partial_{x}^{3} v=G h, \quad v(x, 0)=v_{0}(x) \\
v(0, t)=v(2 \pi, t), \quad \partial_{x} v(0, t)=\partial_{x} v(2 \pi, t), \quad \partial_{x}^{2} v(0,2 \pi)=\partial_{x}^{2} v(2 \pi, t),
\end{array}\right.
$$

and a linear closed loop system associated with (1.15),

$$
\left\{\begin{array}{l}
\partial_{t} v+\partial_{x}^{3} v=-\mathcal{K} G v, \quad v(x, 0)=v_{0}(x) \\
v(0, t)=v(2 \pi, t), \quad \partial_{x} v(0, t)=\partial_{x} v(2 \pi, t), \quad \partial_{x}^{2} v(0,2 \pi)=\partial_{x}^{2} v(2 \pi, t)
\end{array}\right.
$$

It has been shown in [15] that the linear system (1.17) is exactly controllable in the space $L^{2}(0,2 \pi)$ and $H_{p}^{3}(0,2 \pi)$. We present here a more general result: the system $(1.17)$ is exactly controllable in the space $H_{p}^{s}(0,2 \pi)$ for any $s \geq 0$. Moreover we show that there exists a bounded linear operator $\Phi$ from the initial/terminal state pair $v_{0}, v_{1}$, each in the space $H_{p}^{s}(0,2 \pi)$, to the corresponding control $h$ in the space $L^{2}\left(0, T ; H_{p}^{s}(0,2 \pi)\right)$, i.e.,

$$
\left\|\Phi\left(v_{0}, v_{1}\right)\right\|_{L^{2}\left(0, T ; H_{p}^{s}(0,2 \pi)\right)} \leq c\left(\left\|v_{0}\right\|_{s}+\left\|v_{1}\right\|_{s}\right) .
$$

The global exact controllability of the linear system and the estimate (1.16) obtained in this section will play an important role in establishing the exact controllability of the nonlinear system. In addition, we show that the solutions of the linear system (1.18) satisfy an inequality similar to (1.16) without the small amplitude assumption on their initial values. This again is a generalization of the result known in [15].

In Section 3 we demonstrate the exact controllability results for the nonlinear system. Our approach is straightforward; in the global control case, since our control power is strong, the proof of Theorem 1.1 is simple and is mainly based on the exact controllability of the associated linear system and the standard Fredholm Alternative Theorem of functional analysis. In the local control case, using the strongly continuous semigroup $W(t)$ generated by the third order operator $-\partial_{x}^{3}$ in the space $L^{2}(0,2 \pi)$, one can rewrite the nonlinear system $(1.1),(1.2),(1.8)$ in its 
equivalent integral equation form:

$$
u(t)=W(t) u_{0}+\int_{0}^{t} W(t-\tau)(G h)(\tau) d \tau-\int_{0}^{t}\left(u \partial_{x} u\right)(\tau) d \tau
$$

This motivates us to define the map

$$
\Gamma(u):=W(t) u_{0}+\int_{0}^{t} W(t-\tau)\left(G \Phi\left(u_{0}, u_{1}\right)\right)(\tau) d \tau-\int_{0}^{t}\left(u \partial_{x} u\right)(\tau) d \tau,
$$

which, from the definition of the operator $\Phi$ in Section 2 , satisfies $(\Gamma(u))(0)=u_{0}$ and $(\Gamma(u))(T)=u_{1}$. It then remains to be shown that the map $\Gamma$ is a contraction in an appropriate space $X$. The difficulty in this approach is that a smoothing effect needs to be established for the operator

$$
\int_{0}^{t} W(t-\tau)\left(u \partial_{x} u\right)(\tau) d \tau
$$

in order to overcome loss of regularity due to the term $u \partial_{x} u$ and to show that $\Gamma$ is a map from the space $X$ into itself.

In Section 4 we show how the stability result Theorem 1.3 can be established through use of a discrete Lyapounov method, provided appropriate existence, uniqueness, and regularity results for the system (1.15) are available. In Section 5 we prove those results. As a consequence we obtain, for small amplitude solutions, the asymptotic stability results which eluded the authors in [8] and [19]. As in the case of open loop control, no results for large system states are yet available.

\section{The Linear System}

We first consider the associated linear open loop control system

$$
\left\{\begin{array}{l}
\partial_{t} v+\partial_{x}^{3} v=G h, \quad v(x, 0)=v_{0}(x), \\
v(0, t)=v(2 \pi, t), \quad \partial_{x} v(0, t)=\partial_{x} v(2 \pi, t), \quad \partial_{x}^{2} v(0, t)=\partial_{x}^{2} v(2 \pi, t),
\end{array}\right.
$$

where the operator $G$ is defined by (1.8) and $h$ is the applied control function.

Let $A$ denote the operator

$$
A w=-w^{\prime \prime \prime}
$$

on the domain $\mathcal{D}(A)=H_{p}^{3}(0,2 \pi)$. A generates a strongly continuous group $W(t)$ on the space $L^{2}(0,2 \pi)$; the eigenfunctions are simply the orthonormal Fourier basis functions in $L^{2}(0,2 \pi)$,

$$
\phi_{k}(x)=\frac{1}{\sqrt{2 \pi}} e^{i k x}, \quad k=0, \pm 1, \pm 2, \ldots
$$

We have the following exact controllability result for the system (2.1).

Theorem 2.1. Let $T>0$ and $s \geq 0$ be given. Then for any $v_{0}, v_{1} \in H_{p}^{s}(0,2 \pi)$ with $\left[v_{0}\right]=\left[v_{1}\right]$, there exists a control function $h \in L^{2}\left([0, T] ; H_{p}^{s}(0,2 \pi)\right)$ such that the equation

$$
\partial_{t} v+\partial_{x}^{3} v=G h
$$

has a solution $v \in C\left([0, T] ; H_{p}^{s}(0,2 \pi)\right)$ satisfying

$$
v(x, 0) \equiv v_{0}(x), \quad v(x, T) \equiv v_{1}(x) .
$$


Proof. It is familiar that the operator $A$, as defined in (2.2), has eigenvalues

$$
\lambda_{k}=-i k^{3},
$$

corresponding to eigenfunctions $\phi_{k}(x)$ for $-\infty<k<\infty$. Relative to the basis $\phi_{k},-\infty<k<\infty$, the initial state $v_{0}(x)$ and the terminal state $v_{1}(x)$ have the expansions, convergent in $H_{p}^{s}(0,2 \pi)$,

$$
v_{j}=\sum_{k=-\infty}^{+\infty} v_{k, j} \phi_{k}, \quad v_{k, j}=\int_{0}^{2 \pi} v_{j}(x) \overline{\phi_{k}(x)} d x
$$

for $j=0,1$.

The homogeneous (adjoint) equation is

$$
\partial_{t} u+\partial_{x}^{3} u=0, \quad x \in(0,2 \pi), t \in R,
$$

also with periodic boundary conditions; it has corresponding solutions

$$
u_{k}(x, t)=e^{\lambda_{k} t} \phi_{k}(x) .
$$

For smooth $h$, periodic on $(0,2 \pi)$, we readily compute, using integration by parts with $v$ satisfying (2.1), that

$$
\frac{d}{d t} \int_{0}^{2 \pi} v(x, t) \overline{u_{k}(x, t)} d x=\int_{0}^{2 \pi}(G h) v(x, t) \overline{u_{k}(x, t)} d x .
$$

Integrating with respect to $t$, we have

$$
\begin{aligned}
\int_{0}^{2 \pi} & v(x, T) \overline{u_{k}(x, T)} d x-\int_{0}^{2 \pi} v(x, 0) \overline{u_{k}(x, 0)} d x \\
= & \int_{0}^{T} \int_{0}^{2 \pi}(G h) v(x, t) \overline{u_{k}(x, t)} d x d t .
\end{aligned}
$$

Continuity considerations then show that the identity (2.6) continues to be valid for $h \in L^{2}\left([0, T] ; H_{p}^{s}(0,2 \pi)\right)$ for $s \geq 0$. Evaluation of the integrals in (2.6) with

$$
\tilde{v}_{k}=\int_{0}^{2 \pi} v(x, T) \overline{\phi_{k}(x)} d x
$$

and $u_{k}$ as in (2.6) shows that

$$
\tilde{v}_{k}-v_{k, 0} e^{\lambda_{k} T}=\int_{0}^{T} e^{\lambda_{k}(T-\tau)} \int_{0}^{2 \pi}(G h)(x, t) \overline{\phi_{k}(x)} d x d t
$$

for $k=0, \pm 1, \ldots$

If we define $p_{k}(t)=e^{\lambda_{k} t}$, then $\mathcal{P} \equiv\left\{p_{k} \mid-\infty<k<\infty\right\}$ may be seen, from the result in [4], to form a Riesz basis for its closed span, $\mathcal{P}_{T}$, in $L^{2}(0, T)$. We let $\mathcal{Q} \equiv\left\{q_{k} \mid-\infty<k<\infty\right\}$ be the unique dual Riesz basis for $\mathcal{P}$ in $\mathcal{P}_{T}$ such that

$$
\int_{0}^{T} q_{j}(t) \overline{p_{k}(t)} d t=\delta_{k j}, \quad-\infty<j, k<\infty .
$$

We take the control $h$ in (2.1) to have the form

$$
h(x, t)=\sum_{j=-\infty}^{+\infty} h_{j} q_{j}(t)\left(G \phi_{j}\right)(x),
$$


where the coefficients $h_{j}$ are to be determined so that, among other things, the series (2.10) is appropriately convergent. Substituting (2.10) into (2.8) yields, using the biorthogonality (2.9),

$$
\begin{aligned}
\tilde{v}_{k} & -v_{k, 0} e^{\lambda_{k} T}=e^{\lambda_{k} T} \sum_{j=-\infty}^{+\infty} h_{j} \int_{0}^{T} e^{\overline{\lambda_{k} t}} q_{j}(t) \int_{0}^{2 \pi} G\left(G \phi_{j}\right)(x) \overline{\phi_{k}(x)} d x d t \\
& =h_{k} e^{\lambda_{k} T} \int_{0}^{2 \pi} G\left(G \phi_{k}\right)(x) \overline{\phi_{k}(x)} d x
\end{aligned}
$$

for $-\infty<k<\infty$. We verify easily that $G$ is a self-adjoint operator in $L^{2}(0,2 \pi)$ such that

$$
\int_{0}^{2 \pi} G\left(G \phi_{k}\right)(x) \overline{\phi_{k}(x)} d x=\left\|G \phi_{k}\right\|_{L^{2}(0,2 \pi)}^{2}, \quad-\infty<k<\infty .
$$

Since $\left|\phi_{k}(x)\right| \equiv \frac{1}{2 \pi}$, for $-\infty<k<\infty$, we have

$$
\begin{aligned}
\left\|G \phi_{k}\right\|_{L^{2}(0,2 \pi)}^{2}=\int_{0}^{2 \pi}\left|g(x)\left(\phi_{k}(x)-\int_{0}^{2 \pi} g(s) \phi_{k}(s) d s\right)\right|^{2} d x \\
=\frac{1}{4 \pi^{2}} \int_{0}^{2 \pi} g(x)^{2} d x-2\left|\int_{0}^{2 \pi} g(x) \phi_{k}(x) d x\right|^{2} \\
\quad+\int_{0}^{2 \pi} g(x)^{2} d x \cdot\left|\int_{0}^{2 \pi} g(x) \phi_{k}(x) d x\right|^{2} \\
:=\beta_{k} .
\end{aligned}
$$

Since $\phi_{0}(x) \equiv \frac{1}{2 \pi}$, it is easy to see, using (2.3), that $\beta_{0}=0$. The fact that $g(x)$ is real valued shows that $g(x) \phi_{k}(x)$ cannot be a constant multiple of $g(x)$ on any interval; thus the first identity above shows that $\beta_{k} \neq 0, k>0$. The familiar Lebesgue lemma together with the second identity above shows that

$$
\lim _{k \rightarrow \infty} \beta_{k}=\int_{0}^{2 \pi} g(x)^{2} d x \neq 0 .
$$

Clearly $\tilde{v}_{0}$ must be zero, since $\beta_{0}=0$. From (2.12) and the fact that $\beta_{k} \neq 0$ for $k \neq 0$ it follows that there is a $\delta>0$ such that

$$
\left|\beta_{k}\right|>\delta \quad \text { for } k \neq 0 \text {. }
$$

Setting $h_{0}=0$ and

$$
h_{k}=\frac{e^{-\lambda_{k} T} v_{k, 1}-v_{k, 0}}{\beta_{k}}, \quad k \neq 0,
$$

we get from (2.11)

$$
\tilde{v}_{k}=v_{k, 1}
$$

where $v_{k, 1}$ is given by (2.3). 
It remains to show that $h$ defined by $(2.13)$ and $(2.10)$ is in $L^{2}\left([0, T] ; H_{p}^{s}(0,2 \pi)\right)$ provided that $v_{0}, v_{1} \in H_{p}^{s}(0,2 \pi)$. To this end, let us write

$$
G \phi_{j}(x)=\sum_{k=-\infty}^{+\infty} a_{j k} \phi_{j}(x)
$$

where

$$
a_{j k}=\int_{0}^{2 \pi} G \phi_{j}(x) \overline{\phi_{k}(x)} d x, \quad-\infty<j, k<\infty .
$$

Thus

$$
h(x, t)=\sum_{j=-\infty}^{+\infty} \sum_{k=-\infty}^{+\infty} h_{j} a_{j k} q_{j}(t) \phi_{k}(x)
$$

and

$$
\begin{aligned}
& \|h\|_{L^{2}\left([0, T] ; H_{p}^{s}(0,2 \pi)\right)}^{2}=\int_{0}^{T} \sum_{k=-\infty}^{+\infty}(1+|k|)^{2 s}\left|\sum_{j=-\infty}^{+\infty} a_{j k} h_{j} q_{j}(t)\right|^{2} d t \\
& \quad=\sum_{k=-\infty}^{+\infty}(1+|k|)^{2 s} \int_{0}^{T}\left|\sum_{j=-\infty}^{+\infty} a_{j k} h_{j} q_{j}(t)\right|^{2} d t \\
& \leq c \sum_{k=-\infty}^{+\infty}(1+|k|)^{2 s} \sum_{j=-\infty}^{+\infty}\left|h_{j}\right|^{2}\left|a_{j k}\right|^{2} \\
& \quad \leq c \sum_{j=-\infty}^{+\infty}\left|h_{j}\right|^{2} \sum_{k=-\infty}^{+\infty}(1+|k|)^{2 s}\left|a_{j k}\right|^{2},
\end{aligned}
$$

where the constant $c$ comes from the Riesz basis property of $\mathcal{Q}$ in $\mathcal{P}_{T}$. However

$$
\begin{aligned}
\left|a_{j k}\right| & =\left|\left(G \phi_{j}, \phi_{k}\right)_{L^{2}(0,2 \pi)}\right| \\
& =\left|\left(g \phi_{j}, \phi_{k}\right)_{L^{2}(0,2 \pi)}-\left(g, \phi_{j}\right)_{L^{2}(0,2 \pi)}\left(g, \phi_{k}\right)_{L^{2}(0,2 \pi)}\right| \\
& =\mid \sum_{m=-\infty}^{+\infty} g_{m}\left(\phi_{m} \phi_{j}, \phi_{k}\right)_{L^{2}(0,2 \pi)} \\
& \quad-\left(\sum_{m=-\infty}^{+\infty} g_{m}\left(\phi_{m}, \phi_{j}\right)_{L^{2}(0,2 \pi)}\right)\left(\sum_{m=-\infty}^{+\infty} g_{m}\left(\phi_{m}, \phi_{k}\right)_{L^{2}(0,2 \pi)}\right) \mid \\
& =\left|\frac{1}{2 \pi} g_{k-j}-g_{j} g_{k}\right| \\
\leq & \frac{1}{2 \pi}\left|g_{k-j}\right|+\left|g_{j}\right|\left|g_{k}\right|,
\end{aligned}
$$


where

$$
g=\sum_{m=-\infty}^{+\infty} g_{m} \phi_{m}
$$

Hence

$$
\left|a_{j k}\right|^{2} \leq c\left(\left|g_{k-j}\right|^{2}+\left|g_{j}\right|^{2}\left|g_{k}\right|^{2}\right)
$$

and

$$
\begin{aligned}
& \sum_{k=-\infty}^{+\infty}(1+|k|)^{2 s}\left|a_{j k}\right|^{2} \leq c \sum_{k=-\infty}^{+\infty}(1+|k|)^{2 s}\left|g_{k-j}\right|^{2}+c \sum_{k=-\infty}^{+\infty}(1+|k|)^{2 s}\left|g_{j}\right|^{2}\left|g_{k}\right|^{2} \\
& \quad \leq c \sum_{k=-\infty}^{+\infty}(1+|k+j|)^{2 s}\left|g_{k}\right|^{2}+c \sum_{k=-\infty}^{+\infty}(1+|k|)^{2 s}\left|g_{j}\right|^{2}\left|g_{k}\right|^{2} \\
& \quad \leq c(1+|j|)^{2 s} \sum_{k=-\infty}^{+\infty}(1+|k|)^{2 s}\left|g_{k}\right|^{2}+c\left|g_{j}\right|^{2} \sum_{k=-\infty}^{+\infty}(1+|k|)^{2 s}\left|g_{k}\right|^{2} \\
& \quad \leq c\left((1+|j|)^{2 s}+\left|g_{j}\right|^{2}\right)\|g\|_{s}^{2} .
\end{aligned}
$$

We have, according to (2.13),

$$
\begin{aligned}
& \|h\|_{L^{2}\left([0, T] ; H_{p}^{s}(0,2 \pi)\right)}^{2} \leq c\left(\sum_{j=-\infty}^{+\infty}\left((1+|j|)^{2 s}+\left|g_{j}\right|^{2}\right)\left|h_{j}\right|^{2}\right)\|g\|_{s}^{2} \\
& \leq c\left(\sum_{j=-\infty}^{+\infty}\left((1+|j|)^{2 s}+\left|g_{j}\right|^{2}\right) \frac{\left|e^{\lambda_{j} T} v_{j, 1}-v_{j, 0}\right|^{2}}{\left|\beta_{j}\right|^{2}}\right)\|g\|_{s}^{2} \\
& \quad \leq c \max _{j \neq 0}\left|\beta_{j}\right|^{-2}\|g\|_{s}^{2} \sum_{j=-\infty}^{+\infty}(1+|j|)^{2 s}\left(\left|v_{j, 1}\right|^{2}+\left|v_{j, 0}\right|^{2}\right) \\
& \leq c \max _{j \neq 0} \frac{1}{\left|\beta_{j}\right|^{2}}\|g\|_{s}^{2}\left(\left\|v_{1}\right\|_{s}^{2}+\left\|v_{0}\right\|_{s}\right) .
\end{aligned}
$$

With this the proof is complete.

Corollary 2.1. Equations (2.10), (2.13) and (2.14) define, for $s \geq 0$, a bounded operator $\Phi$ :

$$
h=\Phi\left(v_{0}, v_{1}\right), \quad \forall v_{0}, v_{1} \in H_{p}^{s}(0,2 \pi),
$$

from $H_{p}^{s}(0,2 \pi) \times H_{p}^{s}(0,2 \pi)$ to $L^{2}\left([0, T] ; H_{p}^{s}(0,2 \pi)\right)$ such that

$$
W(T) v_{0}+\int_{0}^{T} W(T-\tau)\left(G\left(\Phi\left(v_{0}, v_{1}\right)\right)\right)(\cdot, \tau) d \tau=v_{1}
$$

for any $\left(v_{0}, v_{1}\right) \in H_{p}^{s}(0,2 \pi) \times H_{p}^{s}(0,2 \pi)$ and

$$
\left\|\Phi\left(v_{0}, v_{1}\right)\right\|_{L^{2}\left([0, T] ; H_{p}^{s}(0,2 \pi)\right)} \leq c\left(\left\|v_{0}\right\|_{s}+\left\|v_{1}\right\|_{s}\right),
$$

where $c$ depends only on $T$ and $g$. 
Now we turn to consideration of the associated linear closed loop system

$$
\left\{\begin{array}{l}
\partial_{t} v+\partial_{x}^{3} v=-\mathcal{K} G v, \quad v(x, 0)=v_{0}(x), \\
v(0, t)=v(2 \pi, t), \quad \partial_{x} v(0, t)=\partial_{x} v(2 \pi, t), \quad \partial_{x}^{2} v(0,2 \pi)=\partial_{x}^{2} v(2 \pi, t) .
\end{array}\right.
$$

Theorem 2.2. Let $s=0$ or $s \geq 1$ be given. Then for any $v_{0} \in H_{p}^{s}(0,2 \pi)$, the system (2.18) has a unique solution $v \in C\left(R ; H_{p}^{s}(0,2 \pi)\right)$. Moreover there exist positive constants $\gamma_{s}$ and $M_{s}$, depending only on $s\left(\gamma_{s_{1}} \leq \gamma_{s_{2}}\right.$ if $\left.s_{1} \geq s_{2}\right)$, such that

$$
\left\|v(\cdot, t)-\left[v_{0}\right]\right\|_{s} \leq M_{s} e^{-\gamma_{s} t}\left\|v_{0}-\left[v_{0}\right]\right\|_{s} .
$$

for any $v_{0} \in H_{p}^{s}(0,2 \pi)$.

Proof. The existence of the solution $v$ follows from standard semigroup theory (cf. [11]). The estimate (2.19) has been established in [15] when $s=0$.

We first show that the estimate (2.19) holds for $s=1$. Without loss of generality, we assume that $\left[v_{0}\right]=0$ and therefore $[v(t)] \equiv 0$. Let $w=\partial_{x} v$. It solves

$$
\left\{\begin{array}{l}
\partial_{t} w+\partial_{x}^{3} w=-\mathcal{K} G w-\mathcal{K}\left[\partial_{x}, G\right] v, \quad w(x, 0)=v_{0}^{\prime}(x), \\
w(0, t)=w(2 \pi, t), \quad \partial_{x} w(0, t)=\partial_{x} w(2 \pi, t), \quad \partial_{x}^{2} w(0,2 \pi)=\partial_{x}^{2} w(2 \pi, t),
\end{array}\right.
$$

where $\left[\partial_{x}, G\right] \equiv \partial_{x} G-G \partial_{x}$. Hence

$$
w(t)=W_{\mathcal{K}} v_{0}^{\prime}-\mathcal{K} \int_{0}^{t} W_{\mathcal{K}}(t-\tau)\left(\left[\partial_{x}, G\right] v\right)(\tau) d \tau,
$$

where $W_{\mathcal{K}}(t)$ is the semigroup generated by the operator $A+\mathcal{K} G$ in the space $L^{2}(0,2 \pi)$.

Note that the mean values of $v_{0}^{\prime}$ and $\left[\partial_{x}, G\right] v$ over the interval $(0,2 \pi)$ are both zero. Using the estimate (2.19) with $s=0$, one obtains

$$
\|w(t)\|_{0} \leq M_{0} e^{-\gamma_{0} t}\left\|v_{0}^{\prime}\right\|_{0}+\mathcal{K} M_{0} \int_{0}^{t} e^{-\gamma_{0}(t-\tau)}\left\|\left[\partial_{x}, G\right] v\right\|_{0}(\tau) d \tau .
$$

Direct computation shows that

$$
\left[\partial_{x} G\right] v=g^{\prime} v-g[v(\cdot, t)]_{g}-g[v(\cdot, t)]_{g^{\prime}},
$$

where $[v(\cdot, t)]_{g} \equiv \int_{0}^{2 \pi} g(y) v(y, t) d y$. Using the estimate (2.19) yields

$$
\left\|\left[\partial_{x}, G\right] v\right\|_{0} \leq c\|v\|_{0} \leq c M_{0} e^{-\gamma_{0} t}\left\|v_{0}\right\|_{0}
$$

for some constant $c>0$ depending only on the function $g$. Turning back to (2.21), we have

$$
\begin{aligned}
& \|w(t)\|_{0} \leq M_{0} e^{-\gamma_{0} t}\left\|v_{0}^{\prime}\right\|_{0}+c \mathcal{K} M_{0} \int_{0}^{t} e^{-\gamma_{0}(t-\tau)} e^{-\gamma_{0} \tau} d \tau\left\|v_{0}\right\|_{0} \\
& \leq M_{0} e^{-\gamma_{0} t}\left\|v_{0}^{\prime}\right\|_{0}+c \mathcal{K} M_{0} t e^{-\gamma_{0} t} .
\end{aligned}
$$

Combining (2.19) with $s=0$ and (2.22) yields the estimate (2.19) with $s=1$.

To show the estimate (2.19) is true for $s>1$ we need the following technical lemma. 
Lemma 2.1. Let $D^{s}$ represent the fractional derivative of order $s$ :

$$
D^{s} u=\sum_{k=-\infty}^{+\infty} u_{k}|k|^{s} e^{i k x}
$$

for any $u \in H_{p}^{s}(0,2 \pi)$. Define

$$
\left[D^{s}, G\right] u=D^{s}(G u)-G\left(D^{s} u\right)
$$

for any $u \in H_{p}^{s}(0,2 \pi)$. Then for any $s>1$, one has

$$
\left\|\left[D^{s}, G\right] u\right\|_{0} \leq c\left(s,\|g\|_{2}\right)\|u\|_{\max \{1, s-1\}} .
$$

Supposing, as we will show later, that the lemma is true, we establish the estimate (2.19) by induction. Assume that (2.19) is true for $1 \leq s \leq s_{0}$. We show that (2.19) is true for $s_{1}=s_{0}+1$. To this end, let $w=D^{s_{1}}$. It solves (2.20) with the operator $\partial_{x}$ replaced by $D^{s_{1}}$, and we have the following estimate:

$$
\|w(t)\|_{0} \leq M_{0} e^{-\gamma_{0} t}\left\|v_{0}^{\prime}\right\|_{0}+\mathcal{K} M_{0} \int_{0}^{t} e^{-\gamma_{0}(t-\tau)}\left\|\left[D^{s_{1}}, G\right] v\right\|_{0}(\tau) d \tau .
$$

Then from (2.23) we have the estimate (2.19) with $1 \leq s \leq s_{0}$, so that

$$
\begin{aligned}
\|w(t)\|_{0} & \leq M_{0} e^{-\gamma_{0} t}\left\|D^{s_{1}} v_{0}\right\|_{0}+c \mathcal{K} M_{s_{0}} \int_{0}^{t} e^{-\gamma_{0}(t-\tau)} e^{-\gamma_{s_{0}} \tau} d \tau\left\|v_{0}\right\|_{0} \\
& \leq M_{0} e^{-\gamma_{0} t}\left\|D^{s_{1}} u_{0}\right\|_{0}+c \mathcal{K} M_{0} t e^{-\gamma_{s_{0}} t} .
\end{aligned}
$$

Together with the estimate (2.19) for $s=0,(2.25)$ implies that (2.19) is true for $s=s_{1}$. By induction we have proved the estimate (2.19) is true for $s \geq 2$. The case $1<s<2$ is proved similarly.

Finally we give a proof of Lemma 2.1 in order to complete the proof of Theorem 2.2 .

To show (2.23), we note that

$$
\left[D^{s}, G\right] v=D^{s}(g v)-g D^{s} v-[v]_{g} D^{s} g+g\left[D^{s} v\right] .
$$

Using [17, Lemma 1.1] with $r=1$, we get

$$
\left\|D^{s}(g v)-g D^{s} v\right\|_{0} \leq c\left(\|g\|_{s}\|v\|_{1}+\|g\|_{2}\|v\|_{\max \{1, s-1\}}\right) .
$$

In addition,

$$
\left\|[v]_{g} D^{s} g\right\|_{0} \leq c\|v\|_{0}
$$

and

$$
\left\|g\left[D^{s} v\right]_{g}\right\|_{0} \leq c\left|\left[D^{s} v\right]_{g}\right| \leq\left. c \sum_{\mid=-\infty}^{+\infty} g_{j}|| j\right|^{s}\left|v_{j}\right| \leq c\|v\|_{\max \{1, s-1\}}
$$

Consequently, the estimate (2.23) follows. 


\section{Exact Controllability}

In this section we prove the exact controllability results for the open loop nonlinear control system:

$$
\left\{\begin{array}{l}
\partial_{t} u+u \partial_{x} u+\partial_{x}^{3} u=G h, \quad u(x, 0)=u_{0}(x), \\
u(0, t)=u(2 \pi, t), \quad \partial_{x} u(0, t)=\partial_{x} u(2 \pi, t), \quad \partial_{x}^{2} u(0,2 \pi)=\partial_{x}^{2} u(2 \pi, t) .
\end{array}\right.
$$

In the global control case, due to the rather strong control power available, not only is the result stronger, but also the proof is simpler. For brevity of notation, when in the sequel we wish to denote a function $u=u(x, t)$ as an element of $L^{2}[0,2 \pi]$, or some related space, we will just write $u(t)$ rather than the more cumbersome $u(\cdot, t)$; this should not result in any confusion.

Proof of Theorem 1.1. According to Theorem 2.1, there exists

$$
h_{1} \in L^{2}\left(0, T ; H_{p}^{s+1}(0,2 \pi)\right)
$$

for which one may find $u \in C\left([0, T] ; H_{p}^{s+1}(0,2 \pi)\right)$ satisfying

$$
\left\{\begin{array}{l}
\partial_{t} u+\partial_{x}^{3} u=G h_{1}, \\
u(x, 0)=u_{0}, \quad u(x, T)=u_{1}(x)
\end{array}\right.
$$

for given $u_{0}, u_{1} \in H_{p}^{s+1}(0,2 \pi)$. Adding $u \partial_{x} u$ to both sides of the above equation, one obtains

$$
\left\{\begin{array}{l}
\partial_{t} u+u \partial_{x} u+\partial_{x}^{3} u=G h_{1}+u \partial_{x} u \\
u(x, 0)=u_{0}, \quad u(x, T)=u_{1}(x)
\end{array}\right.
$$

Thus it suffices to show that there exists $h_{2} \in L^{2}\left(0, T ; H_{p}^{s}(0,2 \pi)\right)$ such that

$$
\left(G h_{2}\right)(x, t)=\left(u \partial_{x} u\right)(x, t)
$$

for all $x \in(0,2 \pi)$ and $t \in(0, T)$.

Note that the equation (3.2), by the definition of the operator $G$, reduces to

$$
h_{2}(x, t)-\frac{1}{2 \pi} \int_{0}^{2 \pi} h_{2}(y, t) g(y) d y=-\frac{1}{g(x)} u(x, t) \partial_{x} u(x, t),
$$

and $u \partial_{x} u / g \in C\left([0, T] ; H_{p}^{s}(0,2 \pi)\right)$ by virtue of the assumption (1.11). Let $Y_{g}^{s}$ be the space $H_{p}^{s}(0,2 \pi)$ endowed with the weighted norm

$$
\|v\|_{Y_{g}^{s}}:=(g v, v)_{H_{p}^{s}(0,2 \pi)}^{\frac{1}{2}} .
$$

For $v \in Y_{g}^{s}$, define

$$
(\tilde{G}(v))(x)=v(x)-\int_{0}^{2 \pi} v(y) g(y) d y
$$

Then the following claim is readily checked.

Claim As an operator from $Y_{g}^{s}$ to $Y_{g}^{s}$,

i. $\tilde{G}$ is a Fredholm operator;

ii. $\tilde{G}^{*}=\tilde{G}$;

iii. $N(\tilde{G})=\{1\}$;

iv. $\tilde{G}$ has a bounded inverse considered as an operator from $Y_{g}^{s} / N(\tilde{G})$ to $Y_{g}^{s}$. 
As a result,

$$
R(\tilde{G})=\overline{R(G)}=N\left(\tilde{G}^{*}\right)^{\perp}=N(\tilde{G})^{\perp} .
$$

Since, for any $t \in(0, T)$,

$$
\left(u \partial_{x} u / g, 1\right)_{Y_{g}^{s}}=\int_{0}^{2 \pi} u(x, t) \partial_{x} u(x, t) d x=0,
$$

one has

$$
u(t) \partial_{x} u(t) / g \in N(\tilde{G})^{\perp}=R(\tilde{G})
$$

for any $t \in(0, T)$. Therefore the equation (3.3) has a solution $h_{2}(., t) \in H_{p}^{s}(0,2 \pi)$ for each fixed $t \in x(0, T)$. The continuous dependence of $h_{2}(\cdot, t)$ on $t$ follows from part iv of the claim. The proof is complete.

In the local control situation, as we pointed out in the introduction, a smoothing property is needed for the operator

$$
v(t)=\int_{0}^{t} W(t-\tau) f(\tau) d \tau
$$

from $f$ to $v$. This needed smoothing property was provided in Bourgain's recent work [3], where he dealt with the Cauchy problem for the periodic KdV equation.

For a function $w:(0,2 \pi) \times R$, define the quantities

$$
\begin{gathered}
\Lambda_{1}(w)=\left(\sum_{n=-\infty}^{\infty}(1+|n|)^{2 s} \int_{-\infty}^{\infty} \frac{|\hat{w}(n, \lambda)|^{2}}{1+\left|\lambda-n^{3}\right|} d \lambda\right)^{1 / 2} \\
\Lambda_{2}(w)=\left(\sum_{n=-\infty}^{\infty}(1+|n|)^{2 s}\left[\int_{-\infty}^{\infty} \frac{|\hat{w}(n, \lambda)|}{1+\left|\lambda-n^{3}\right|} d \lambda\right]^{2}\right)^{1 / 2}
\end{gathered}
$$

and

$$
\Lambda_{3}(w)=\left(\sum_{n=-\infty}^{\infty}(1+|n|)^{2 s} \int_{-\infty}^{\infty}|\hat{w}(n, \lambda)|^{2}\left(1+\left|\lambda-n^{3}\right|\right) d \lambda\right)^{1 / 2}
$$

where $\widehat{w}(n, \lambda)$ denotes the Fourier transform of $w(x, t)$ with respect to both the $x$ and $t$ variables.

Following Bourgain [3], for any $s \geq 0$, we introduce the following space:

$$
X^{s}=\left\{w \in L^{2}\left(R ; H_{p}^{s}(0,2 \pi)\right) ; \Lambda_{3}(w)<\infty\right\}
$$

with the norm $\|v\|_{X^{s}}:=\Lambda_{3}(w)$. It is clear that $X^{s}$ is a Hilbert space.

In the following, the function $\psi(t)$ is assumed to be smooth and satisfy

$$
\psi(t)= \begin{cases}1, & \text { if } t \in\left[-\frac{1}{2}, \frac{1}{2}\right] \\ 0, & \text { if } t \neq(-1,1)\end{cases}
$$

Before we proceed to show the exact controllability result in the local control case, we present the following five technical lemmas, which not only play important roles in the proof of Theorem 1.2, but also provide the basic tools for establishing stabilizability in Section 4 and Section 5. 
Lemma 3.1. For any $h \in X_{s}$, one has

$$
\left\|\psi\left(t / \delta^{-1}\right) h\right\|_{X_{s}} \leq c_{1}(\delta)\|h\|_{X_{s}}
$$

where

$$
c_{1}(\delta)=\left\{\begin{array}{l}
c_{2}(b) \delta^{(1-2 b) / 2} \quad \text { for any } 1 / 2<b \leq 1 \text { and } 0<\delta \leq 1 ; \\
+\infty \quad \text { as } \delta \rightarrow+\infty ;
\end{array}\right.
$$

and $c_{2}(b) \rightarrow+\infty$ as $b \rightarrow 1 / 2^{+}$.

Proof. See Lemma 2.2 of [20].

Lemma 3.2. For any $u_{0} \in H_{p}^{s}(0,2 \pi)$,

$$
\left\|\psi(t / \delta) W(t) u_{0}\right\|_{X_{s}} \leq c_{1}(\delta)\left\|u_{0}\right\|_{s}
$$

Lemma 3.3.

$$
\left\|\psi(t / \delta) \int_{0}^{t} W(t-\tau) f(\tau) d \tau\right\|_{X_{s}} \leq c_{1}(\delta)\left(\Lambda_{1}(f)+\Lambda_{2}(f)\right) .
$$

Lemma 3.4. For any $0<T \leq+\infty$

$$
\sup _{-T<t<+T}\left\|\int_{0}^{t} W(t-\tau) f(\tau) d \tau\right\|_{s} \leq c \Lambda_{2}(f)
$$

where $c>0$ is independent of $T$.

Lemmas 3.2-3.4 are slight modifications of results included in the proof of Theorem 5 in [3] (cf. [20]).

Lemma 3.5. For any $u, v \in X_{s, b}$ with $[u(., t)]=[v(., t)] \equiv 0$,

$$
\Lambda_{1}\left(\psi^{2}(t / \delta) \partial_{x}(u v)\right)+\Lambda_{2}\left(\psi^{2}(t / \delta) \partial_{x}(u v)\right) \leq c \delta^{1 / 12}\|u\|_{X_{s}}\|v\|_{X_{s}},
$$

where $c>0$ is independent of $\delta$.

If $u=v$, the estimate (3.9) is contained in Lemma 7.41 and Lemma 7.42 in [3]. Estimates (3.9) and (3.7) together reflect a smoothing property of the linear KdV equation (1.1) with $\alpha=0$ from the forcing term $f$ to the solution $u$.

Now we are in a position to prove Theorem 1.2.

Proof of Theorem 1.2. Let $W(t)$ be the semigroup generated by the operator $A$, defined by $(2.2)$, on the space $L^{2}(0,2 \pi)$. We rewrite the system (3.1) in its equivalent integral equation form:

$$
u(t)=W(t) u_{0}+\int_{0}^{t} W(t-\tau)(G h)(\tau) d \tau-\int_{0}^{t} W(t-\tau)\left(u \partial_{x} u\right)(\tau) d \tau .
$$

Assuming temporarily that

$$
\left[u_{0}\right]=\left[u_{1}\right]=0,
$$

we define

$$
w(T, u):=\int_{0}^{T} W(T-\tau)\left(u \partial_{x} u\right)(\tau) d \tau .
$$

According to Corollary 2.1, for given $u_{0}, u_{1} \in H_{p}^{s}(0,2 \pi)$, if one chooses

$$
h=\Phi\left(u_{0}, u_{1}+w(T, u)\right)
$$


in the equation (3.10), then

$$
\begin{aligned}
u(t)=W(t) u_{0}+\int_{0}^{t} W(t-\tau)\left(G \Phi\left(u_{0}, u_{1}+w(T, u)\right)\right)(\tau) d \tau \\
-\int_{0}^{t} W(t-\tau)\left(u \partial_{x} u\right)(\tau) d \tau
\end{aligned}
$$

and

$$
u(0)=u_{0}, \quad u(T)=u_{1}
$$

by virtue of the definition of the operator $\Phi$ (cf. Corollary 2.1). This suggests that we consider the map

$$
\begin{aligned}
\Gamma(u)=W(t) u_{0}+\int_{0}^{t} W(t-\tau)\left(G\left(\Phi\left(u_{0}, u_{1}+w(T, u)\right)\right)\right)(\tau) d \tau \\
-\int_{0}^{t} W(t-\tau)\left(u \partial_{x} u\right)(\tau) d \tau .
\end{aligned}
$$

If the map $\Gamma$ is shown to be a contraction in an appropriate space, then its fixed point $u$ is a solution of $(3.1)$ with $h=\Phi\left(u_{0}, u_{1}+w(T, u)\right)$ and satisfies $u(x, T) \equiv$ $u_{1}(x)$. We show this is the case in the space $X_{s}$.

We modify the map $\Gamma$ as follows:

$$
\begin{gathered}
\Gamma(u)=\psi_{1}(t) W(t) u_{0}+\psi_{1}(t) \int_{0}^{t} W(t-\tau) \psi_{2}(\tau)\left(G \Phi\left(u_{0}, u_{1}+w(T, u)\right)\right)(\tau) d \tau \\
-\psi_{1}(t) \int_{0}^{t} \psi_{2}(\tau) W(t-\tau)\left(u \partial_{x} u\right)(\tau) d \tau
\end{gathered}
$$

for any $u \in X_{s}$, where $\psi_{1}(t)$ is a smooth function with its support inside the interval $(-T-1 / 2, T+1 / 2)$ and $\psi_{1}(t)=1$ for $t \in[-T, T]$, and $\psi_{2}(t)$ is a nonnegative smooth function with supp $\psi_{2} \subset(-T-1 / 2, T+1 / 2)$ satisfying $\psi_{2}(t)=1$ for any $t$ in the support of $\psi_{1}$. Applying (3.5)-(3.9) to (3.17) $(\delta=2 T)$ yields

$$
\begin{aligned}
\|\Gamma(u)\|_{X_{s}} \leq & c\left\|u_{0}\right\|_{s}+c \Lambda_{1}\left(\psi_{2}(t) G\left(\Phi\left(u_{0}, u_{1}+w(T, u)\right)\right)\right. \\
& +c \Lambda_{2}\left(\psi_{2}(t) G\left(\Phi\left(u_{0}, u_{1}+w(T, u)\right)\right)\right. \\
& +c \Lambda_{1}\left(\psi_{2}(t)\left(u \partial_{x} u\right)(t)\right)+c \Lambda_{2}\left(\psi_{2}(t)\left(u \partial_{x} u\right)(t)\right) .
\end{aligned}
$$

It then follows from the definitions of $\Lambda_{1}$ and $\Lambda_{2}$ and the operator $\Phi$ that

$$
\begin{gathered}
\Lambda_{1}\left(\psi_{2}(t) G\left(\Phi\left(u_{0}, u_{1}+w(T, u)\right)\right)\right) \leq c\left\|G \Phi\left(u_{0}, u_{1}+w(T, u)\right)\right\|_{s} \\
\leq c\left(\left\|u_{0}\right\|_{s}+\left\|u_{1}\right\|_{s}+\|w(T, u)\|_{s}\right) .
\end{gathered}
$$

Similarly, one obtains

$$
\Lambda_{2}\left(\psi_{2}(t) G\left(\Phi\left(u_{0}, u_{1}+w(T, u)\right)\right)\right) \leq c\left(\left\|u_{0}\right\|_{s}+\left\|u_{1}\right\|_{s}+\|w(T, u)\|_{s}\right) .
$$


Using Lemmas 3.1, 3.2 and 3.3, it follows that

$$
\begin{aligned}
\|w(T, u)\|_{s} & =\left\|\int_{0}^{T} W(T-\tau)\left(u \partial_{x} u\right)(\tau) d \tau\right\|_{s} \\
& \leq \sup _{t \in R}\left\|\psi_{1}(t) \int_{0}^{t} W(t-\tau) \psi_{2}(\tau)\left(u \partial_{x} u\right)(\tau) d \tau\right\|_{s} \\
& \leq \Lambda_{2}\left(\psi_{2}(\tau)\left(u \partial_{x} u\right)(\tau) d \tau\right) \\
& \leq c\|u\|_{X_{s}}^{2}
\end{aligned}
$$

Consequently,

$$
\|\Gamma(u)\|_{X_{s}} \leq c\left(\left\|u_{0}\right\|_{s}+\left\|u_{1}\right\|_{s}\right)+c\|u\|_{X_{s}}^{2} .
$$

For $M>0$, let $S_{M}$ be a bounded subset of $X_{s}$ :

$$
S_{M}=\left\{v \in X_{s} \mid[v]=0,\|v\|_{X_{s}} \leq M\right\} .
$$

Then, for any $u \in S_{M}$,

$$
\|\Gamma(u)\|_{X_{s}} \leq c\left\|u_{0}\right\|_{s}+c\left\|u_{1}\right\|_{s}+c M^{2} .
$$

We choose $\delta>0$ and $M>0$ such that

$$
2 c \delta+c M^{2} \leq M, \quad c M<1 / 2 .
$$

Then,

$$
\|\Gamma(u)\|_{X_{s}} \leq M
$$

for any $u \in S_{M}$, if $\left\|u_{0}\right\|_{s} \leq \delta$ and $\left\|u_{1}\right\|_{s} \leq \delta$. In addition, for any $u, v \in S_{M}$, since

$$
\begin{array}{r}
\Gamma(u)-\Gamma(v)=\psi_{1}(t) \int_{0}^{t} W(t-\tau) \psi_{2}(\tau)(G \Phi(0, w(T, u)-w(T, v)))(\tau) d \tau \\
-\psi_{1}(t) \int_{0}^{t} W(t-\tau) \psi_{2}(\tau)\left(\frac{1}{2} \partial_{x}((u+v)(u-v))\right)(\tau) d \tau
\end{array}
$$

and

$$
w(T, u)-w(T, v)=\int_{0}^{T} W(T-\tau) \frac{1}{2} \partial_{x}((u+v)(u-v))(\tau) d \tau,
$$

a similar argument shows that

$$
\begin{aligned}
\|\Gamma(u)-\Gamma(v)\|_{X_{s}} & \leq \frac{c}{2}\|u+v\|_{X_{s}}\|u-v\|_{X_{s}} \\
& \leq c M\|u-v\|_{X_{s}} \\
& \leq \frac{1}{2}\|u-v\|_{X_{s}} .
\end{aligned}
$$

Thus the map $\Gamma$ is a contraction on $S_{M}$ provided that $\delta$ and $M$ are chosen according to (3.19) and $\left\|u_{0}\right\|_{s} \leq \delta,\left\|u_{1}\right\|_{s} \leq \delta$. As a result, its fixed point $u \in S_{M}$ is a unique solution of the integral equation

$$
\begin{gathered}
u(t)=\psi_{1}(t) W(t) u_{0}+\psi_{1}(t) \int_{0}^{t} W(t-\tau) \psi_{2}(\tau)\left(G\left(\Phi\left(u_{0}, u_{1}+w(T, u)\right)\right)\right)(\tau) d \tau \\
-\psi_{1}(t) \int_{0}^{t} \psi_{2}(\tau) W(t-\tau)\left(u \partial_{x} u\right)(\tau) d \tau
\end{gathered}
$$


for any $t \in R$. In particular, for $t \in[0, T]$,

$$
\begin{aligned}
u(t)=W(t) u_{0}+\int_{0}^{t} W(t-\tau)\left(G\left(\Phi\left(u_{0}, u_{1}+w(T, u)\right)\right)\right)(\tau) d \tau \\
-\int_{0}^{t} W(t-\tau)\left(u \partial_{x} u\right)(\tau) d \tau .
\end{aligned}
$$

That is to say, $u \in C\left([0, T] ; H_{p}^{s}(0,2 \pi)\right)$ solves

$$
\left\{\begin{array}{l}
\partial_{t} u+u \partial_{x} u+\partial_{x}^{3} u=G \Phi\left(u_{0}, u_{1}+w(T, u)\right), \\
u(x, 0)=u_{0}(x), \quad u(x, T)=u_{1}(x)
\end{array}\right.
$$

for $t \in[0, T]$.

Here we need to point out a detail which is missing from the above argument; in order for the map $\Gamma(u)$ to make sense it is necessary to be certain that $w(T, u)$ is well defined, which is true, of course, if $w(t, u)$ is continuous in $H_{p}^{s}$ as a function of $t$. To this end, let us suppose that $u \in X_{s}$. Since Lemma 3.5 shows that

$$
\left(\Lambda_{1}+\Lambda_{2}\right)\left(\psi(t / \delta) \partial_{x}\left(v^{2}\right)\right) \leq c\|v\|_{X_{s}}^{2},
$$

it is clearly enough to show that if $\left(\Lambda_{1}+\Lambda_{2}\right)(f)$ is bounded, then

$$
w_{f}(t) \equiv \int_{0}^{t} W(t-\tau) f(\cdot, \tau) d \tau
$$

is continuous in $H_{p}^{s}$ as a function of $t$. It is a standard exercise to demonstrate this if $f \in \mathcal{H}_{s} \equiv L^{2}\left(R^{1} ; H_{p}^{s}\right)$; we omit the details. Let us denote by $\mathcal{H}_{\Lambda}$ the completion of $\mathcal{H}_{s}$ relative to the norm $\|f\|_{\Lambda} \equiv\left(\Lambda_{1}+\Lambda_{2}\right)(f)$, noting that the sum of a norm and a seminorm is, indeed, a norm. Then $\mathcal{H}_{\Lambda}$ is a Banach space which includes $\mathcal{H}_{s}$ as a dense subspace relative to \|\|$_{\Lambda}$; i.e., given $f$ with $\|f\|_{\Lambda}$ bounded, we can find a sequence $\left\{f_{k}\right\} \subset \mathcal{H}_{s}$ converging to $f$ relative to \|\|$_{\Lambda}$. Using Lemma 3.4 we see that the corresponding functions $w_{f_{k}}(\cdot, t)$ converge uniformly to $w_{f}(\cdot, t)$ relative to \|\|$_{s}$ on finite $t$ intervals. It follows that $w_{f}(\cdot, t)$ is continuous in $H_{p}^{s}$ as a function of $t$. This is all that is needed to show that $w(T, u)$ is well defined in the argument presented above. Since the other terms in (3.17) cause no problem in regard to continuity, it also follows from this that the controlled solution $u$, i.e., the fixed point of $\Gamma$, is continuous in $H_{p}^{s}$ as a function of $t$, so that the sense in which the endpoints of the control problem are achieved is not ambiguous. This is an important point because it is not in general true that functions in $X_{s}$ are continuous in $H_{p}^{s}$ as functions of $t$.

To summarize, we may choose the control

$$
h=\Phi\left(u_{0}, u_{1}+w(T, u)\right)
$$

to guide the corresponding solution $u(t)$ from $u(0)=u_{0}$ to $u(T)=u_{1}$, provided the assumption (3.11) is satisfied. In the general case wherein that assumption is not valid, the 'volume' $[u(., t)]$ continues to be conserved, as we have seen in the introduction:

$$
[u(., t)]=\left[u_{0}\right]=\left[u_{1}\right]:=\beta, \quad \forall t \in[0, T] .
$$

Since the above proof is strongly based on Lemma 3.3, which requires that $[u(., t)] \equiv$ 0 , it does not cover the general case $\beta \neq 0$. However, the basic idea of the proof in the general case is the same as in the case we have just treated; it suffices merely to indicate the needed modifications. 
Let $u$ be a solution of (3.1) with $[u(., t)] \equiv\left[u_{0}\right]=\beta$, and let $v(x, t)=u(x, t)-\beta$. It is easily seen that $v(x, t)$ solves

$$
\left\{\begin{array}{l}
\partial_{t} v+v \partial_{x} v+\partial_{x}^{3} v+\beta \partial_{x} v=G h, \quad v(x, 0)=u_{0}(x)-\beta:=v_{0}(x), \\
v(0, t)=v(2 \pi, t), \quad \partial_{x} v(0, t)=\partial_{x} v(2 \pi, t), \quad \partial_{x}^{2} v(0, t)=\partial_{x}^{2} v(2 \pi, t) .
\end{array}\right.
$$

As before we first consider the associated linear system

$$
\left\{\begin{array}{l}
\partial_{t} w+\partial_{x}^{3} w+\beta \partial_{x} w=G h, \quad w(x, 0)=u_{0}(x)-\beta:=w_{0}(x) \\
w(0, t)=w(2 \pi, t), \quad \partial_{x} w(0, t)=\partial_{x} w(2 \pi, t), \quad \partial_{x}^{2} w(0, t)=\partial_{x}^{2} w(2 \pi, t) .
\end{array}\right.
$$

The same proof as given for Theorem 2.1 shows that the system (3.21) possesses the identical exact controllability properties.

Passing to the nonlinear system (3.20), we let $W_{\beta}(t)$ be the semigroup generated by the operator

$$
A_{\beta}=-\partial_{x}^{3}-\beta \partial_{x}
$$

with $\mathcal{D}\left(A_{\beta}\right)=H_{p}^{3}(0,2 \pi)$ in the space $L^{2}(0,2 \pi)$. Then (3.20) is equivalent to

$$
v(t)=W_{\beta}(t) v_{0}+\int_{0}^{t} W_{\beta}(t-\tau)(G h)(\tau) d \tau-\int_{0}^{t} W_{\beta}(t-\tau)\left(v \partial_{x} v\right)(\tau) d \tau .
$$

The proof is the same as before except that we consider the map $\Gamma$ in the space

$$
\begin{aligned}
X_{s}^{\beta}:= & \left\{v \in L^{2}\left(R: H_{p}^{s}(0,2 \pi)\right) ;\right. \\
& \left.\left(\sum_{n=-\infty}^{+\infty}(1+|n|)^{2 s} \int_{-\infty}^{\infty}\left(1+\left|\lambda-n^{3}-\beta n\right|\right)|\hat{v}(n, \lambda)|^{2} d \lambda\right)^{1 / 2}<\infty\right\}
\end{aligned}
$$

instead of the space $X_{s}$. Also, we need the extended definitions

$$
\begin{gathered}
\Lambda_{1}^{\beta}(w)=\left(\sum_{n=-\infty}^{\infty}(1+|n|)^{2 s} \int_{-\infty}^{\infty} \frac{|\hat{w}(n, \lambda)|^{2}}{1+\left|\lambda-n^{3}-\beta n\right|} d \lambda\right)^{1 / 2}, \\
\Lambda_{2}^{\beta}(w)=\left(\sum_{n=-\infty}^{\infty}(1+|n|)^{2 s}\left[\int_{-\infty}^{\infty} \frac{|\hat{w}(n, \lambda)|}{1+\left|\lambda-n^{3}-\beta n\right|} d \lambda\right]^{2}\right)^{1 / 2} .
\end{gathered}
$$

The needed Lemmas 3.1, 3.2 and 3.3 still hold with the spaces $X_{s}, \Lambda_{1}$ and $\Lambda_{2}$ replaced by the spaces $X_{s}^{\beta}, \Lambda_{1}^{\beta}$ and $\Lambda_{2}^{\beta}$, respectively (see [3] and [20]). The proof may thus be considered complete.

Remark 3.1. Professor J. L. Bona, in a private communication, has pointed out that the fact that this controllability result is valid on arbitrarily short time intervals is counterintuitive in the context of soliton solutions of the KdV equation; such solitons originating some distance from the control region, i.e., the support of the function $g(x)$, could not be affected significantly by the indicated control mechanism until some time had elapsed. This is a valid, and valuable, observation; it indicates that the results obtained here for small solutions (these would not, in general, include soliton solutions on a periodic domain) probably cannot be extended 
without some additional condition on the control interval to obtain control results for solutions of arbitrary amplitude; the present results are only valid for solutions small enough in amplitude so that the KdV equation continues to behave in much the same way as the third order linear dispersion equation described in Section 2.

\section{Asymptotic Stability}

Having established, in the proof of Theorem 1.2 in Section 3, an exact controllability result for the system (3.1) valid for "local" control and small initial and terminal states, it is reasonable to conjecture that, again for small initial states, the asymptotic stability results which eluded the authors in [19] and [8] might now be within reach. We will see that this is, indeed, the case but, because Bourgain's smoothing results, as we have seen, are valid only for bounded time intervals, we cannot proceed with use of the continuous time infinite dimensional Lyapounov approach as in the corresponding work with "point dissipation" in [16]. Instead, we find it necessary to work with the discrete Lyapounov theory and discrete time intervals $[(k-1) T, k T], k=1,2,3, \ldots$, where $T>0$ is as specified below.

Let $H$ be a separable Hilbert space and let $\mathcal{A}$ be a bounded operator on $H$. We will assume that $\mathcal{A}$ is a stability operator in the discrete sense, i.e., there is a positive number $M$ and a real number $r$ satisfying $0<r<1$ such that, using $\|\cdot\|_{H}$ interchangeably to denote the norm in $H$ or in $\mathcal{L}(H, H)$ as required,

$$
\left\|\mathcal{A}^{k}\right\|_{H}<M r^{k}, \quad k=0,1,2, \ldots
$$

Thus for solution sequences $\left\{x_{k}\right\}$ of the linear recursion equation

$$
x_{k+1}=\mathcal{A} x_{k}, \quad k=0,1,2, \ldots,
$$

with initial states $x_{0}$ given in $H$, we have the estimate

$$
\|x\|_{H} \leq M r^{k}\left\|x_{0}\right\|_{H}, \quad k=0,1,2, \ldots
$$

Consider then in $\mathcal{L}(H, H)$ the operator equation

$$
X=\mathcal{A}^{*} X \mathcal{A}+W,
$$

where $W$ is a given positive definite self-adjoint operator on $H$ and $\mathcal{A}^{*}$ denotes the adjoint operator of $\mathcal{A}$. Multiplying (4.3) on the left by $\left(\mathcal{A}^{*}\right)^{k}$ and on the right by $\mathcal{A}^{k}, k=0,1,2, \ldots$, transposing the term on the left to the right for $k=1,2,3, \ldots$, and adding the resulting equations, we have, for any positive $n$,

$$
X=\left(\mathcal{A}^{*}\right)^{n+1} X \mathcal{A}^{n}+\sum_{k=0}^{n}\left(\mathcal{A}^{*}\right)^{k} W \mathcal{A}^{k} .
$$

From (4.1) we see that the indicated sum converges and the operator $I_{n}^{-1}$, where

$$
I_{n}(X)=X-\left(\mathcal{A}^{*}\right)^{n} X \mathcal{A}^{n},
$$

converges to the identity operator in $\mathcal{L}(H, H)$ as $n \rightarrow \infty$. Thus we have for (4.3) the unique solution

$$
X=\sum_{k=0}^{\infty}\left(\mathcal{A}^{*}\right)^{k} W \mathcal{A}^{k}
$$

and it is easy to see that $X \in \mathcal{L}(H, H)$ is positive definite and self-adjoint. Conversely, if we have a positive definite self-adjoint solution $X$ of (4.3), use of $\mathcal{A}^{k+1}=$ $\mathcal{A} \mathcal{A}^{k}, \quad k=0,1,2, \ldots$, together with the form of (4.3) shows that

$$
\left(\mathcal{A}^{*}\right)^{k+1} X \mathcal{A}^{K+1}=\left(\mathcal{A}^{*}\right)^{k} \mathcal{A}^{*} X \mathcal{A} \mathcal{A}^{k}=\left(\mathcal{A}^{*}\right)^{k}(X-W) \mathcal{A}^{k},
$$


from which, since $0<X-W<X$ (in the sense of quadratic forms on $H$ ), an estimate of the form (4.1) readily follows, along with the estimate

$$
\left\|x_{k}\right\|_{H} \leq M r^{k}\left\|x_{0}\right\|_{H}, \quad k=0,1,2, \ldots,
$$

valid for solutions $\left\{x_{k}\right\}$ of (4.2).

If we generalize (4.2) to a nonlinear recursion equation

$$
x_{k+1}=\mathcal{A} x_{k}+q\left(x_{k}\right), \quad k=0,1,2, \ldots,
$$

where $q: H \rightarrow H$ is continuous and satisfies

$$
\lim _{\|x\|_{H} \rightarrow 0}\|q(x)\|_{H} /\|x\|_{H}=0,
$$

we can use the framework just developed to show that solutions of (4.4) with small initial states $x_{0} \in H$ also tend exponentially to 0 as $k \rightarrow \infty$. For we have (using the convenient notation $\langle x, y\rangle=y^{*} x$, where $\langle\cdot, \cdot\rangle$ is the inner product in $H$ )

$$
\begin{aligned}
& \left(x_{k+1}\right)^{*} X x_{k+1}=\left(\mathcal{A} x_{k}+g\left(x_{k}\right)\right)^{*} X\left(\mathcal{A} x_{k}+q\left(x_{k}\right)\right) \\
& \quad=\left(x_{k}\right)^{*} \mathcal{A}^{*} X \mathcal{A} x_{k}+\left(x_{k}\right)^{*} X q\left(x_{k}\right)+q\left(x_{k}\right)^{*} X x_{k}+q\left(x_{k}\right)^{*} X q\left(x_{k}\right) .
\end{aligned}
$$

Using (4.5) and (4.6) together with the assumed positivity of $X$, we can see that if $\left\|x_{k}\right\|_{H}$ is sufficiently small we will have

$$
\left(x_{k+1}\right)^{*} X x_{k+1} \leq\left(x_{k}\right)^{*}((1-\epsilon) X-W) x_{k}
$$

for $\epsilon$ positive and sufficiently small, in particular small enough so that $0<$ $(1-\epsilon) X-W<\rho X$ for some $\rho \in[0,1)$. Thus

$$
\left(x_{k+1}\right)^{*} X x_{k+1} \leq \rho\left(x_{k}\right)^{*} X x_{k}, \quad k=0,1,2, \ldots,
$$

and we conclude that $x_{k} \rightarrow 0$ at a uniform exponential rate as claimed. We will use a variant of this approach in the work to follow to the closed loop system (1.15).

Now let $A$ be defined as in (2.2) and $G$ as in (1.8). In [15] we have described the feedback control system wherein the external input, or control, $f$, appearing in (1.1), is given by

$$
f(x, t)=-\mathcal{K}(G u)(x, t), \quad \mathcal{K}>0 .
$$

The linear version of (1.1), corresponding to $\alpha=0$, takes in the Hilbert space $H_{p}^{s}(0,2 \pi)(s \geq 0)$ the abstract form

$$
\frac{d u}{d t}=(A-\mathcal{K} G) u
$$

solutions $u(t)$ corresponding to a given initial state $u_{0} \in H_{p}^{s}(0,2 \pi)$ being given for $t \geq 0$ by

$$
u(\cdot, t)=W_{\mathcal{K}}(t) u_{0},
$$

where $W_{\mathcal{K}}(t)$ is the strongly continuous semigroup of bounded operators on $H_{p}^{s}(0,2 \pi)$ generated by the operator $A-\mathcal{K} G$. Since $G$ is a bounded operator, the existence of $W_{\mathcal{K}}(t)$ follows immediately from that of $W(t)$ (cf. [11]). Because $A$ and $G$ annihilate the one-dimensional subspace of constant states (functions) in $H_{p}^{s}(0,2 \pi)$, so does $A-\mathcal{K} G$, and, as a consequence, $W_{\mathcal{K}}(t)$ leaves the space $H_{p, 0}^{s}$, which may be defined as either the orthogonal complement of the subspace $C$ of constant functions in $H_{p}^{s}(0,2 \pi)$, or, equally well, as $H_{p, 0}^{s} \equiv H_{p}^{s}(0,2 \pi) / C$, invariant. We will continue to use $A-\mathcal{K} G$ and $W_{\mathcal{K}}(t)$ to denote the restrictions of those operators to $\mathcal{D}(A) \cap H_{p, 0}^{s}$ and $H_{p, 0}^{s}$ respectively; there should be no resulting confusion. 
In Section 2, we have shown that $W_{\mathcal{K}}(t)$ has uniform exponential decay to 0 in the space $H_{p, 0}^{s}$; there are positive constants $M$ and $\gamma_{0}$ such that

$$
\left\|W_{\mathcal{K}}(t)\right\|_{s, 0} \leq M e^{-\gamma_{0} t}, \quad t>0
$$

Here we use $\|\cdot\|_{s, 0}$ to denote either the uniform operator norm in $\mathcal{L}\left(H_{p, 0}^{s}, H_{p, 0}^{s}\right)$ or the vector norm in that space $H_{p, 0}^{s}:\|u\|_{s, 0}=\|u-[u]\|_{s}$.

Let us fix $T>0$ and define

$$
\gamma=e^{-\gamma_{0} T} ; \quad 0<\gamma<1
$$

Also defining

$$
\mathcal{A}_{\mathcal{K}}=W_{\mathcal{K}}(T)
$$

we have the inequality (4.1) with $\mathcal{A}$ replaced by $\mathcal{A}_{\mathcal{K}}$, provided we restrict $\mathcal{A}_{\mathcal{K}}$ to $H_{p, 0}^{s}$ as just discussed, which we assume henceforth. It follows that there is a unique positive definite self-adjoint solution $X_{\mathcal{K}} \in \mathcal{L}\left(H_{p, 0}^{s}, H_{p, 0}^{s}\right)$ of the discrete Lyapounov equation

$$
X_{\mathcal{K}}=\left(\mathcal{A}_{\mathcal{K}}\right)^{*} X_{\mathcal{K}} \mathcal{A K}+I_{s}
$$

where $I_{s}$ denotes the identity operator on $H_{p, 0}^{s}$.

In the case of solutions $u=u(x, t)$ of (1.15) the identity (4.4) is replaced by

$$
u(t)=W_{\mathcal{K}}(t) u_{0}-\int_{0}^{t} W_{\mathcal{K}}(t-\tau)\left(u u_{x}\right)(\tau) d \tau,
$$

and a similar identity obtains on later intervals,

$$
u\left(t+t_{0}\right)=W_{\mathcal{K}}(t) u\left(t_{0}\right)-\int_{0}^{t} W(t-\tau)\left(u u_{x}\right)\left(\tau+t_{0}\right) d \tau,
$$

provided existence and adequate regularity have been established on $\left[0, t+t_{0}\right]$. Since the dynamics are time invariant, we can write (4.4) in the form

$$
u\left(t+t_{0}\right)=W_{\mathcal{K}}(t) u\left(t_{0}\right)+q_{\mathcal{K}}\left(t, u\left(t_{0}\right)\right) .
$$

Now let $T>0$ be fixed as in (4.11) and $s \geq 0$ be given. Suppose we can show that, for $\psi \in H_{p, 0}^{s}, \quad q_{\mathcal{K}}(T, \psi)$ is well defined and:

i) $q_{\mathcal{K}}(T, \psi)=q_{\mathcal{K}}(\cdot, T, \psi) \in H_{p, 0}^{s}$;

ii) Given any $\epsilon>0$, we can find $\delta>0$ such that for $\|\psi\|_{s, 0}<\delta$ we have

$$
\left\|q_{\mathcal{K}}(T, \psi)\right\|_{s, 0}<\epsilon\|\psi\|_{s, 0}
$$

iii) There exists an $\eta>0$ such that if $u_{0} \in H_{p, 0}^{s}$ with $\left\|u_{0}\right\|_{s, 0} \leq \eta$, then the corresponding solution $u$ of (1.15) satisfies

$$
\sup _{0 \leq t \leq T}\|u(\cdot, t)\|_{s, 0} \leq c\left\|u_{0}\right\|_{s, 0},
$$

where $c>0$ is independent of $u_{0}$.

If this can be shown to be the case, we can repeat the argument of (4.4)-(4.8) more or less word for word, replacing $x_{k}$ by $u(k T), k=0,1,2, \ldots, \mathcal{A}$ by $\mathcal{A}_{\mathcal{K}}, X$ by $X_{\mathcal{K}}$ and $q\left(x_{k}\right)$ by $q_{\mathcal{K}}(T, u(k T))$ to obtain an inequality, for $0<\rho<1$,

$$
u((k+1) T)^{*} X_{\mathcal{K}} u((k+1) T) \leq \rho u(k T)^{*} X_{\mathcal{K}} u(k T), \quad k=0,1,2, \ldots,
$$

provided $\|u(0)\|_{s, 0}=\left\|u_{0}\right\|_{s, 0}$ is sufficiently small. From this, we see that there exists an $M>0$ such that

$$
\|u(k T)\|_{s, 0} \leq M \rho^{k}\left\|u_{0}\right\|_{s, 0}
$$


for any $k \geq 0$. Since $\rho<1$, there exists a $k_{0}>0$ such that if $k>k_{0}$, one would have

$$
\|u(k T)\|_{s, 0} \leq \eta
$$

where $\eta$ is as given in iii). Consequently, for $k>k_{0}$,

$$
\sup _{k T \leq t \leq(k+1) T}\|u(\cdot, t)\|_{s, 0} \leq c M \rho^{k}\left\|u_{0}\right\|_{s, 0}
$$

where $c>0$ is independent of $k$. From this we clearly see that $\|u(t)\|_{s, 0}$ has uniform exponential decay to 0 as $t \rightarrow \infty$, provided $\left\|u\left(t_{0}\right)\right\|_{s, 0}=\left\|u_{0}\right\|_{s, 0}$ is sufficiently small.

In the next section we will prove i), ii) and iii) in the context of general results on well-posedness of generalized solutions of the closed loop system (1.15), in a sense to be made specific there, and the relationship between those solutions and solutions of the corresponding linear equation with the same initial conditions. That work, together with the discussion presented above, will provide the proof of Theorem 1.3.

\section{Well-Posedness of the Closed Loop System}

In this section we consider the well-posedness problem for the closed loop system

$$
\left\{\begin{array}{l}
\partial_{t} u+\alpha u \partial_{x} u+\partial_{x}^{3} u+\mathcal{K} G u=0, \quad u(x, 0)=u_{0}(x) \\
u(0, t)=u(2 \pi, t), \quad \partial_{x} u(0, t)=\partial_{x} u(2 \pi, t), \quad \partial_{x}^{2} u(0,2 \pi)=\partial_{x}^{2} u(2 \pi, t),
\end{array}\right.
$$

and provide a proof for the statements i), ii) and iii) in the previous section.

In the sequel we adhere to the notational conventions introduced in [20] and Section 3 of this paper; in particular we define $\Lambda_{k}, k=1,2,3$, as in Section 3 and we let the space $X_{s}$ be defined as in (3.4) with norm $\|w\|_{X_{s}}=\Lambda_{3}(w), s \geq 0$.

The first major result of this section is the following theorem on existence, uniqueness and smoothness of solutions.

Theorem 5.1. Let $s \geq 0$ be given. There is a positive valued function $T=T(r)$, $r>0$, with possible infinite values, nonincreasing with increasing $r$, such that, given an initial state $u_{0} \in H_{p}^{s}(0,2 \pi)$ with $\left\|u_{0}\right\|_{s} \leq r$, the system (5.1) has a unique solution $u \in C\left([-T(r), T(r)] ; H_{p}^{s}(0,2 \pi)\right)$, which is a restriction of a function $\tilde{u}$ in the space $L^{2}\left(R ; H_{p}^{s}(0,2 \pi)\right)$ satisfying

$$
\|u\|_{X_{s}}<\infty
$$

Preparatory to the proof of this result, we introduce three lemmas which will be cited in the proof itself.

Lemma 5.1. The group of bounded operators $W_{\mathcal{K}}(t)$ satisfies, relative to the original group $W(t)$, the relationship

$$
W_{\mathcal{K}}(t) u_{0}=W(t) u_{0}-\mathcal{K} \int_{0}^{t} W(t-\tau) G\left(W_{\mathcal{K}}(\tau) u_{0}\right) d \tau u
$$

for any $u_{0} \in L^{2}(0,2 \pi)$. Further, for $f \in L^{2}\left(R ; L^{2}(0,2 \pi)\right)$,

$$
\begin{aligned}
\int_{0}^{t} W_{\mathcal{K}}(t & -\tau) f(\tau) d \tau=\int_{0}^{t} W(t-\tau) f(\tau) d \tau \\
& -\mathcal{K} \int_{0}^{t} W(t-\tau) G\left(\int_{0}^{\tau} W_{\mathcal{K}}(\tau-r) f(r) d r\right) d \tau .
\end{aligned}
$$


Proof. Let $u_{0} \in \mathcal{D}(A)=\mathcal{D}(A-\mathcal{K} G)$ and $u(t)=W_{\mathcal{K}}(t) u_{0}$. It is easy to see that

$$
\partial_{t} u(t)=A u(t)-\mathcal{K} G u(t)=A u(t)-\mathcal{K} G\left(W_{\mathcal{K}}(t) u_{0}\right) .
$$

Applying the "variation of parameters" formula, we have (5.2) for $u_{0}$ as specified. The result for general $u_{0} \in L^{2}(0,2 \pi)$ then follows by continuity. The result (5.3) is proved similarly. The proof is complete.

Since all equations (5.1) with $\alpha>0$ are equivalent under rescaling of the variable $u$, it will be enough to consider the case corresponding to $\alpha=1$. The most natural approach, using the strongly continuous group of bounded operators $W(t)$, would be to replace (5.1) by the integral equation

$$
u(t)=W(t) u_{0}-\int_{0}^{t} W(t-\tau)\left(\left(u \partial_{x} u\right)(\tau)+\mathcal{K} G u(\tau)\right) d \tau ;
$$

indeed, in those cases wherein $u$ does not have enough regularity for (5.1) to be satisfied in the strict sense, the term "solution of (5.1)" must be understood in the sense of this integral equation. However, when one attempts to use a fixed point approach based directly on this equation it turns out that we must assume $\mathcal{K}$ small in order to obtain the contraction property. Seeking to avoid this unnecessary restriction, one is next led to use the group $W_{\mathcal{K}}(t)$ in a somewhat non-standard way, as indicated in

Lemma 5.2. If $u$ is a solution of (5.1) such that $u \partial_{x} u \in L^{2}\left(R ; L^{2}(0,2 \pi)\right)$, then $u(t)$ satisfies the integral equation

$$
\begin{aligned}
u(t)= & W_{\mathcal{K}}(t) u_{0}-\int_{0}^{t} W(t-\tau)\left(u \partial_{x} u\right)(\tau) d \tau \\
& -\mathcal{K} \int_{0}^{t} W_{\mathcal{K}}(t-\tau) G \int_{0}^{\tau} W(\tau-r)\left(u \partial_{x} u\right)(r) d r d \tau .
\end{aligned}
$$

Proof. Using the notation of the semigroup $W_{\mathcal{K}}(t)$, we can rewrite the system (5.1) in the following integral equation form:

$$
u(t)=W_{\mathcal{K}}(t) u_{0}-\int_{0}^{t} W_{\mathcal{K}}(t-\tau)\left(u \partial_{x} u\right)(\tau) d \tau
$$

Then (5.5) follows from (5.6) by using (5.3) with $f=u \partial_{x} u$. The proof is complete.

Once the identity of (5.4) and (5.5) is established for $u \partial_{x} u$ as described, the corresponding identity with much weaker assumptions on that function follows from continuity considerations. Thus we may also define weak solutions of (5.1) to be solutions of the integral equation (5.5).

With respect to the last term occurring in (5.5) we have the result expressed in

Lemma 5.3. Let $s \geq 0$ and $T>0$ be given. Let $\phi$ and $\theta$ be smooth nonnegative functions on $R$ with compact support, identically equal to 1 on $[-T, T]$. For $v \in$ $X_{s}$ with $[v(\cdot, t)] \equiv 0$, define $(c f .(5.7))$

$$
\tilde{u}(t)=\int_{0}^{t} W_{\mathcal{K}}(t-\tau) G \int_{0}^{\tau} W(\tau-r)\left(v \partial_{x} v\right)(r) d r
$$


assuming the function $g(x)$ in terms of which $G$ is defined $(c f .(1.8))$ lies in $C^{s}[0,2 \pi]$, and

$$
\hat{u}(t)=\phi(t) \int_{0}^{t} W_{\mathcal{K}}(t-\tau) G \int_{0}^{\tau} W(\tau-r) \theta(r)\left(v \partial_{x} v(r)\right) d r .
$$

Then $\tilde{u} \in C\left([-T, T] ; H_{p}^{s}(0,2 \pi)\right)$ and, for $\tilde{c}=\tilde{c}(T)>0$,

$$
\sup _{-T \leq t \leq T}\|\tilde{u}(t)\|_{s}<\tilde{c}\|v\|_{X^{s}}^{2} .
$$

Further, $\hat{u}$ agrees with $\tilde{u}(t)$ for $t \in[-T, T]$, lies in $X_{s}$ and, for $\hat{c}>0$, independent of $v$, we have

$$
\|\hat{u}\|_{X_{s}} \leq \hat{c}\|v\|_{X_{s}}^{2}
$$

Proof. From work already carried out in the proof of Theorem 1.2 we see that

$$
\int_{0}^{\tau} W(\tau-r)\left(v \partial_{x} v\right)(r) d r
$$

lies in $C\left([-T, T] ; H_{p}^{s}(0,2 \pi)\right)$ if $v \in X_{s}$ with $[v(\cdot, t)] \equiv 0$, its supremum $H_{p}^{s}(0,2 \pi)$ norm on any finite interval being bounded in terms of $\|v\|_{X_{s}}^{2}$. Then, if the function $g(x)$ in the definition of $G$ is smooth as indicated, it will also be true that

$$
h(t) \equiv G \int_{0}^{\tau} W(\tau-r) \theta(r)\left(v \partial_{x} v(r)\right) d r
$$

lies in $C\left([-T, T] ; H_{p}^{s}(0,2 \pi)\right)$. Then for

$$
\tilde{u}(t)=\int_{0}^{t} W_{\mathcal{K}}(t-\tau) h(\tau) d \tau
$$

the standard arguments show that $\tilde{u}(t) \in C\left([-T, T] ; H_{p}^{s}(0,2 \pi)\right)$ and that we have a bound of the form (5.9). To show that $\hat{u} \in X_{s}$ we note that, since $W_{\mathcal{K}}(t)$ is the semigroup generated by $A-\mathcal{K} G$, it is quite direct to see that, with

$$
\hat{h}(t) \equiv G \int_{0}^{\tau} W(\tau-r) \theta(r) v \partial_{x} v(r) d r,
$$

we have

$$
\hat{u}(t)=\phi(t) \int_{0}^{t} W(t-\tau)[\hat{h}(\tau)-\mathcal{K} G \hat{u}(\tau)] d \tau .
$$

Since $\hat{h}(t)$ and $\mathcal{K} G \hat{u}(\tau) \in C\left([-T, T] ; H_{p}^{s}(0,2 \pi)\right), \theta(\tau)(h(\tau)-\mathcal{K}(G \tilde{u})(\tau)) \in \mathcal{H}_{s}$ and therefore $\mathcal{H}_{\Lambda}$, these spaces being defined just as in the proof of Theorem 1.2 in Section 3. Then Lemma 3.3 applies to show that $\hat{u}$ lies in $X_{s}$ with norm in that space bounded by the norm of $\hat{h}-\mathcal{K} G \hat{u}$ in the space $\mathcal{H}_{\Lambda}$, which, in turn, is bounded in terms of $\|v\|_{X_{s}}^{2}$, completing the proof.

Proof of Theorem 5.1. For simplicity, we assume that $\left[u_{0}\right]=0$. The general case can be proved similarly (cf. [3] or the proof of Theorem 1.2 in Section 3).

Let $v \in X_{s}$ with $[v(\cdot, t)] \equiv 0$; define

$$
\begin{gathered}
\left(F_{1}(v)\right)(t)=\int_{0}^{t} W(t-\tau) \theta(\tau / \delta)\left(v \partial_{x} v\right)(\tau) d \tau, \\
\left(F_{2}(v)\right)(t)=\mathcal{K} \int_{0}^{t} W(t-\tau) \theta(\tau / \delta)\left(G F_{1}(v)\right)(\tau) d \tau,
\end{gathered}
$$




$$
\begin{gathered}
\left(F_{G}(v)\right)(t)=G\left[\int_{0}^{t} W(t-\tau)\left(G F_{1}(v)\right)(\tau) d \tau\right], \\
\left(F_{\mathcal{K}}(v)\right)(t)=\mathcal{K}^{2} \int_{0}^{t} W(t-\tau) \theta(\tau / \delta)\left(F_{G}(v)\right)(\tau) d \tau, \\
\left(I_{\mathcal{K}}\left(u_{0}\right)\right)(t)=\mathcal{K} \int_{0}^{t} W(t-\tau) \theta(\tau / \delta) G W_{\mathcal{K}}(\tau) u_{0} d \tau .
\end{gathered}
$$

Then consider the mapping $w=\Gamma(v)$, where

$$
\Gamma(v) \equiv \phi(t / \delta)\left[W(t) u-I_{\mathcal{K}}\left(u_{0}\right)-F_{1}(v)+F_{2}(v)-F_{\mathcal{K}}(v)\right] .
$$

Inspection shows that the equation $u=\Gamma(u)$ is equivalent to the integral equation (5.5) for $-\frac{\delta}{2} \leq t \leq \frac{\delta}{2}$.

From the properties of $W_{\mathcal{K}}(t)$ as a strongly continuous semigroup on $H_{p}^{s}(0,2 \pi)$, $s \geq 0$, we obtain for any positive $T$ the uniform bounds

$$
\begin{gathered}
\left\|W_{\mathcal{K}}(t) u_{0}\right\|_{s} \leq c\left\|u_{0}\right\|_{s}, \quad-T \leq t \leq T \\
\left\|\int_{0}^{t} W_{\mathcal{K}}(t-\tau) f(\tau) d \tau\right\|_{s} \leq c \sup _{-T \leq t \leq T}\|f(\cdot, t)\|_{s}, \quad-T \leq t \leq T .
\end{gathered}
$$

Assume $0<\delta \leq 1$. Using these with Lemmas 3.1-3.5 we obtain the following estimates.

From Lemma 3.3:

$$
\left\|\phi(\cdot / \delta) W(\cdot)\left(u_{0}\right)\right\|_{X_{s}} \leq c\|u\|_{s} .
$$

From Lemma 3.4 (noting that $\Lambda_{2}(f) \leq\|f\|_{L^{2}\left(R ; H_{p}^{s}(0,2 \pi)\right)}$ ), the boundedness of $G$ and (5.11):

$$
\begin{aligned}
& \left\|\phi(\cdot / \delta) I_{\mathcal{K}}\left(u_{0}\right)\right\|_{X_{s}} \leq c(b) \delta^{\frac{1-2 b}{2}}\left[\int_{-\infty}^{\infty}|\theta(t / \delta)|^{2}\left\|G W_{\mathcal{K}}(t) u_{0}\right\|_{s} d t\right]^{\frac{1}{2}} \\
& \quad \leq c(b) \delta^{\frac{1-2 b}{2}} \delta^{\frac{1}{2}} \sup _{-\delta \leq t \leq \delta}\left\|G W_{\mathcal{K}}(t) u_{0}\right\|_{s} \leq c(b) \delta^{1-b}\left\|u_{0}\right\|_{s} .
\end{aligned}
$$

From Lemmas 3.1 and 3.4:

$$
\left\|v(\cdot / \delta) F_{1}(v)\right\|_{X_{s}} \leq c(b) \delta^{\frac{7-12 b}{2}}\|v\|_{X_{s}}^{2}
$$

From Lemmas 3.1, 3.4 and 3.5:

$$
\begin{aligned}
& \left\|\phi(\cdot / \delta) F_{2}(v)\right\|_{X_{s}} \leq c(b) \delta^{\frac{1-2 b}{2}}\left[\int_{-\infty}^{\infty}|\theta(\tau / \delta)|^{2}\left\|G F_{1}(v)\right\|_{s}^{2} d \tau\right]^{\frac{1}{2}} \\
& \quad \leq c(b) \delta^{\frac{1-2 b}{2}} \delta^{\frac{1}{2}} \sup _{-\delta \leq t \leq \delta}\left\|G F_{1}(v)\right\|_{s} \leq c(b) \delta^{(13 / 12)-b}\|v\|_{X_{s}}^{2} .
\end{aligned}
$$

Finally in this list, from Lemma 3.4, (5.11) and (5.12):

$$
\begin{aligned}
& \left\|\phi(\cdot / \delta) F_{\mathcal{K}}(v)\right\|_{X_{s}} \leq c(b) \delta^{\frac{1-2 b}{2}}\left[\int_{-\infty}^{\infty}|\theta(t / \delta)|^{2}\left\|F_{G}(v)(\cdot, t)\right\|_{s} d t\right]^{\frac{1}{2}} \\
& \leq c(b) \delta^{\frac{1-2 b}{2}} \delta^{1 / 2} \sup _{-\delta \leq t \leq \delta}\left\|F_{1}(v)\right\|_{s} \\
& \leq c(b) \delta^{\frac{1-2 b}{2}} \delta^{1 / 2} \sup _{-\delta \leq t \leq \delta}\left\|F_{1}(v)\right\|_{s} \leq c(b) \delta^{(13 / 12)-b}\|v\|_{X_{s}}^{2} .
\end{aligned}
$$


Combining (5.14)-(5.17) with the form of $\Gamma$ in (5.16) and choosing $\epsilon_{0}$ so that (recalling $b>1 / 2$ )

we have (cf. (5.16))

$$
\epsilon_{0} \equiv \frac{7-12 b}{12} \leq \frac{1}{12}
$$

$$
\left\|\Gamma(v)-W_{\mathcal{K}}(t) u_{0}\right\|_{X_{s}} \leq c \delta^{\epsilon_{0}}\|v\|_{X_{s}}^{2}
$$

so that

$$
\|\Gamma(v)\|_{X_{s}} \leq c\left\|u_{0}\right\|_{s}+c \delta^{\epsilon_{0}}\|v\|_{X_{s}} .
$$

Let

$$
D=\left\{v \in X_{s} ;[v(\cdot, t)] \equiv 0,\|v\|_{X_{s}} \leq 2 c\left\|u_{0}\right\|_{s}\right\}
$$

and let $\delta \in(0,1)$ be chosen small enough so that

$$
4 c^{2}\|u\|_{s} \delta^{\epsilon_{0}} \leq 1 .
$$

Then for any $v \in D,(5.19)$ shows that $\|G(v)\|_{X_{s}} \leq 2 c\left\|u_{0}\right\|_{s}$ and thus $\Gamma(D) \subset D$. A similar computation allows us to see that

$$
\left\|\Gamma\left(v_{1}\right)-\Gamma\left(v_{2}\right)\right\|_{X_{s}} \leq \frac{1}{2}\left\|v_{1}-v_{2}\right\|_{X_{s}}, \quad v_{1}, v_{2} \in D .
$$

As a consequence, $\Gamma$ has a unique fixed point $u \in D$ which is a solution of the integral equation (5.5) and thus a generalized solution of (5.1) for $-\delta / 2 \leq t \leq \delta / 2$. We take $T=\delta / 2$. The conclusion that $u \in C\left([-T, T], H_{p}^{s}(0,2 \pi)\right)$ follows from application of Proposition 5.3 to the third term on the right hand side of (5.5), noting that the comparable result for the second term there has already been obtained as part of the proof of Theorem 1.2 in Section 3; the first term on the right hand side of (5.12) clearly causes no difficulty. This completes the proof.

In the above proof of Theorem 5.1, if we withdraw the assumption $0<\delta \leq 1$, we would have

$$
\|\Gamma(v)\|_{X_{s}} \leq c\left\|u_{0}\right\|_{s}+\xi(\delta)\|v\|_{X_{s}}^{2},
$$

where $\xi(\delta)$ is a positive continuous function with $\xi \rightarrow \infty$ as $\delta$ tends to $\infty$. For fixed $\delta>0$, let $\eta=\xi^{-1}(\delta)$. If $\left\|u_{0}\right\|_{s} \leq \eta$, then $\|\Gamma(v)\|_{X_{s}} \leq 2 c\left\|u_{0}\right\|_{s}$. The map $\Gamma$ is still a contraction mapping on the ball $D$. Consequently, we have the following:

Corollary 5.1. Let $s \geq 0$ and $T>0$ be given. There exist positive numbers $\eta$ and $M$ such that for any $u_{0} \in H_{p}^{s}(0,2 \pi)$ with $\left\|u_{0}\right\|_{s} \leq \eta$, the corresponding solution $u$ of the system (5.1) satisfies

$$
\sup _{-T \leq t \leq T}\|u(t)\|_{s} \leq M\left\|u_{0}\right\|_{s} .
$$

If $s=0,1$ or $s \geq 2$, then solutions of the system (5.1) have the following a priori estimate:

$$
\sup _{[-T, T]}\|u(\cdot, t)\|_{s} \leq \xi_{1}\left(\left\|u_{0}\right\|_{s}, T\right)
$$

where $\xi_{1}: R^{+} \times R^{+} \rightarrow R^{+}$is a nondecreasing continuous function (see [8]). Then a standard argument gives us the following global result.

Corollary 5.2. Let $s=0,1$ or $s \geq 2$ and $T>0$ be given. Then for any $u_{0} \in$ $H_{p}^{s}(0,2 \pi)$, the system (5.1) has a unique solution $u \in C\left([-T, T] ; H_{p}^{s}(0,2 \pi)\right)$ which is a restriction on $[-T, T]$ of a function $\tilde{u} \in X_{s}$. 
The second principal theorem concerns the continuity of the solution $u$ with respect to the initial state $u$ and the relationship between solutions of (5.1) in general (w.l.o.g. $\alpha=1$ ) and solutions of the linear equation ((5.1) with $\alpha=0)$.

Theorem 5.2. Using the same notation as in the statement of Theorem 5.1, if $\|u\|_{s}<r$ and $T^{\prime}<T(r)$, there is a neighborhood $\mathcal{U}$ of $u_{0}$ in $H_{p}^{s}(0,2 \pi)$ such that if $v_{0} \in \mathcal{U}$ and $\left[v_{0}\right]=\left[u_{0}\right]$, the solution $v(t)$ corresponding to the initial state $v_{0}$ is defined for $t \in\left[-T^{\prime}, T^{\prime}\right]$ and satisfies

$$
\sup _{-T^{\prime} \leq t \leq T^{\prime}}\|v(t)-u(t)\|_{s} \leq \beta\left\|v_{0}-u_{0}\right\|_{s},
$$

where $\beta=\beta(r)>0$ depends only on $r$. Further, if

$$
w(t)=W_{\mathcal{K}}(t) u_{0}
$$

is the solution of the corresponding linear system with the same initial state $u_{0}$ with $\left\|u_{0}\right\|_{s} \leq r, r$ sufficiently small, then

$$
\sup _{-T(r) \leq t \leq T(r)}\|w(t)-u(t)\|_{s} \leq \hat{\beta}\left\|u_{0}\right\|_{s}^{2},
$$

where $\hat{\beta}=\hat{\beta}(r)$ depends only on $r$.

Proof. The continuity of the solution $u$ on the interval $[-T(r), T(r)]$ obtained in Theorem 5.1, together with examination of the proof of that theorem, allows us to see that if instead of defining $\Gamma$ on $D$ as in (5.10), (5.20) we had instead defined $\Gamma$ on

$$
\begin{array}{r}
\hat{D}=\left\{v \in X_{s} \cap C\left([-T(r), T(r)] ; H_{p}^{s}(0,2 \pi)\right) ;[v(\cdot, t)] \equiv 0,\right. \\
\left.\|v\|_{X_{s}}+\sup _{-T(r) \leq t \leq T(r)}\|v(\cdot, t)\|_{s} \leq 2 c\left\|u_{0}\right\|_{s}\right\}
\end{array}
$$

using

$$
\left\|v_{1}-v_{2}\right\|_{X_{s}}+\sup _{-T(r) \leq t \leq T(r)}\left\|v_{1}-v_{2}\right\|_{s} \equiv \hat{\rho}\left(v_{1}, v_{2}\right)
$$

as the metric on $\hat{D}$, we would have obtained the invariance of $\hat{D}$ and the contraction property of $\Gamma$ on $\hat{D}$ in much the same way as before. Taking $\mathcal{U}$ to be a sufficiently small neighborhood of $u_{0}$ and $v_{0} \in \mathcal{U}$ and defining a modified map $\tilde{\Gamma}$ just as in (5.10), with $u_{0}$ replaced by $v_{0}$, and then replacing $T(r)$ of Theorem 5.1 by $T^{\prime}<T(r)$, we see for $\mathcal{U}$ sufficiently small that $\hat{D}$ is invariant under both $\Gamma$ and $\tilde{\Gamma}$ and there exists a common contraction constant $\hat{\gamma}, 0 \leq \hat{\gamma}<1$, applicable to both maps, i.e.,

$$
\hat{\rho}\left(\Gamma\left(v_{1}\right), \Gamma\left(v_{2}\right)\right)<\hat{\gamma} \hat{\rho}\left(v_{1}, v_{2}\right), \quad \hat{\rho}\left(\tilde{\Gamma}\left(v_{1}\right), \tilde{\Gamma}\left(v_{2}\right)\right)<\hat{\gamma} \hat{\rho}\left(v_{1}, v_{2}\right),
$$

for $v_{1}, v_{2} \in \hat{D}$. From the form of the first term on the right hand side of (5.5) it is clear that, for given $w \in \hat{D}$, there is a positive number $\tilde{\beta}(r)$ such that

$$
\hat{\rho}(\Gamma(w), \tilde{\Gamma}(w)) \leq \hat{\beta}(r)\left\|u_{0}-v_{0}\right\|_{s} .
$$

Let $v$ be the fixed point of $\tilde{\Gamma}$; the standard proof of the contraction fixed point theorem shows that for any $v_{1} \in \hat{D}$ the sequence $\left\{v_{k} \mid k \leq 1\right\}$ generated by $v_{k+1}=$ 
$\tilde{\Gamma}\left(v_{k}\right), k=1,2,3, \ldots$, converges to $v$ relative to the metric $\hat{\rho}$, and we have

$$
\hat{\rho}\left(v, v_{1}\right) \leq \frac{1}{1-\gamma} \hat{\rho}\left(v_{2}, v_{1}\right) .
$$

Taking $v_{1}=u,(5.25)$ and (5.26) give

$$
\hat{\rho}(v, u) \leq \frac{1}{1-\gamma} \hat{\rho}(\tilde{\Gamma}(u), u)=\frac{1}{1-\gamma} \hat{\rho}(\tilde{\Gamma}(u), \Gamma(u)) \leq \frac{\tilde{\beta}(r)}{1-\gamma}\left\|u_{0}-v_{0}\right\|_{s} .
$$

As $\gamma$ also depends only on $r$, we have (5.25) with $\beta(r)=\tilde{\beta}(r) /(1-\gamma)$.

The result (5.23) is obtained in much the same way, except that we need only refer to the map $\Gamma$ as originally defined with respect to the interval $[-T(r), T(r)]$, but again using the metric $\hat{\rho}(u, v)$. We start the fixed point iteration with

$$
v_{1}(t) \equiv W_{\mathcal{K}}(t) u_{0}
$$

and note that, with the modified metric, the counterpart of (5.25) will yield

$$
\hat{\rho}\left(\Gamma(v), W_{\mathcal{K}}(t) u_{0}\right) \leq c \delta^{\epsilon_{0}}\|v\|_{X_{s}}^{2} .
$$

Then, from the definition of $\hat{D}$, we have (with a different $c$ )

$$
\hat{\rho}\left(v_{2}, v_{1}\right)=\hat{\rho}\left(\Gamma(v), W_{\mathcal{K}}(t) u_{0}\right) \leq c \delta^{\epsilon_{0}}\left\|u_{0}\right\|_{s}^{2} .
$$

Then we obtain (5.26) and, taking (5.27),(5.28) into account,

$$
\hat{\rho}(v, w) \leq \frac{c}{1-\gamma} \delta^{\epsilon_{0}}\left\|u_{0}\right\|_{s}^{2}
$$

from which (5.23) follows, and the proof is complete.

Finally, with regard to the application of these results to the proof of Theorem 1.3, we note that since (cf. i), ii), iii) near the end of Section 4) the function

$$
q_{\mathcal{K}}\left(t, u_{0}\right)=u(t)-W_{\mathcal{K}}(t) u_{0}
$$

is, again from Theorem 5.1, continuous in $H_{p}^{s}(0,2 \pi)$ as a function of $t$, we have the first conclusion of i) as indicated. The second conclusion follows, after a continuity argument, from

$$
\int_{0}^{2 \pi} v \partial_{x} v d x=0
$$

Finally, condition ii) follows from (5.23) of Theorem 5.2, and condition iii) follows from Corollary 5.1.

\section{REFERENCES}

[1] J. L. Bona and R. Smith, The initial value problem for the Korteweg-de Vries equation, Philos. Trans. Roy. Soc. London A, 278 (1975), 555 - 601. MR 52:6219

[2] J. Bourgain, Fourier transform restriction phenomena for certain lattice subsets and applications to non-linear evolution equations, part I: Schrödinger equations, Geometric and Functional Analysis 3 (1993), 107 - 156. MR 95d:35160a

[3] J. Bourgain, Fourier transform restriction phenomena for certain lattice subsets and applications to non-linear evolution equations, part II: the KdV equation, Geometric and Functional Analysis, 3 (1993), 209 - 262. MR 95d:35160b

[4] A. E. Ingham, Some trigonometrical inequalities with application to the theory of series, Math. A., 41 (1936), $367-379$.

[5] T. Kato, On the Cauchy problem for the (generalized) Korteweg-de Vries equations, Advances in Mathematics Supplementary Studies, Studies in Applied Math., 8 (1983), 93 - 128. MR 86f: 35160 
[6] C. E. Kenig, G. Ponce and L. Vega, The Cauchy problem for the Korteweg-de Vries equation in Sobolev spaces of negative indices, Duke Math. J. 71 (1993), 1-21. MR 94g:35196

[7] V. Komornik, D. L. Russell and B. -Y. Zhang, Stabilisation de l'equation de Korteweg-de Vries, C. R. Acad. Sci. Paris, t. 312(1991), 841 - 843. MR 92b:35134

[8] V. Komornik, D. L. Russell and B. -Y. Zhang, Control and stabilization of the Korteweg-de Vries equation on a periodic domain, submitted to J. Differential Equations.

[9] R. M. Miura, The Korteweg-de Vries equation: a survey of results, SIAM Review, 18 (1976), 412 - 459. MR 53:8689; MR 57:6908

[10] R. M. Miura, C. S. Gardner and M. D. Kruskal, Korteweg-de Vries equations and generalizations II: Existence of conservation laws and constants of motion, J. Math. Physics, 9 (1968), 1204 - 1209. MR 40:6042b

[11] A. Pazy, Semigroups of Linear Operators and Applications to Partial Differential Equations, Applied Mathematical Sciences, Vol. 44, Springer-Verlag, 1983. MR 85g:47061

[12] Lord Rayleigh, On waves, Phil. Mag., 1 (1876), 257 - 279.

[13] D. L. Russell, Computational study of the Korteweg-de Vries equation with localized control action, Distributed Parameter Control Systems: New Trends and Applications, G. Chen, E. B. Lee, W. Littman, and L. Markus, eds., Lecture Notes in Pure and Appl. Math., vol. 128, Marcel Dekker, New York, 1991, 195-203. MR 92c:65113

[14] D. L. Russell, Controllability and stabilizability theory for linear partial differential equations: Recent progress and open questions, SIAM Rev., 20 (1978), 639 - 739. MR 80c:93032

[15] D. L. Russell and B. -Y. Zhang, Controllability and stabilizability of the third-order linear dispersion equation on a periodic domain, SIAM J. Control and Optimization, 31 (1993), 659 - 676. MR 94g:93018

[16] D. L. Russell and B. -Y. Zhang, Smoothing and decay properties of solutions of the Kortewegde Vries equation on a periodic domain with point dissipation, J. Math. Anal. Appl. 190 (1995), 449-488. MR 95k:35180

[17] J. C. Saut and R. Temam, Remarks on the Korteweg-de Vries equation, Israel J. Math., 24(1976), 78 - 87. MR 56:12676

[18] R. Temam, Sur un problème non linéaire, J. Math. Pures Appl., 48 (1969), 159 - 172. MR 41:5799

[19] B. -Y. Zhang, Some results for nonlinear dispersive wave equations with applications to control, Ph. D thesis, University of Wisconsin-Madison, 1990.

[20] B. - Y. Zhang, A remark on the Cauchy problem for the Korteweg-de Vries equation on a periodic domain, Differential and Integral Equations 8 (1995), 1191-1204. MR 96a:35182

Department of Mathematics, Virginia Polytechnic Institute and State University, BlACKSBURG, ViRginia 24061-123

E-mail address: russell@math.vt.edu

Department of Mathematical Sciences, University of Cincinnati, Cincinnati, Ohio 45221

E-mail address: bzhang@math.uc.edu 